\title{
Robust Linear MIMO in the Downlink: A Worst-Case Optimization with Ellipsoidal Uncertainty Regions
}

\author{
Gan Zheng, ${ }^{1}$ Kai-Kit Wong, ${ }^{1}$ and Tung-Sang Ng ${ }^{2}$ \\ ${ }^{1}$ Adastral Park Campus, Martlesham Heath, University College London, Suffolk IP5 3RE, UK \\ ${ }^{2}$ Telecommunications Research Group, The University of Hong Kong, Pokfulam Road, Hong Kong
}

Correspondence should be addressed to Kai-Kit Wong, k.wong@adastral.ucl.ac.uk

Received 8 February 2008; Accepted 26 June 2008

Recommended by Geert Leus

This paper addresses the joint robust power control and beamforming design of a linear multiuser multiple-input multiple-output (MIMO) antenna system in the downlink where users are subjected to individual signal-to-interference-plus-noise ratio (SINR) requirements, and the channel state information at the transmitter (CSIT) with its uncertainty characterized by an ellipsoidal region. The objective is to minimize the overall transmit power while guaranteeing the users' SINR constraints for every channel instantiation by designing the joint transmitreceive beamforming vectors robust to the channel uncertainty. This paper first investigates a multiuser MISO system (i.e., MIMO with single-antenna receivers) and by imposing the constraints on an SINR lower bound, a robust solution is obtained in a way similar to that with perfect CSI. We then present a reformulation of the robust optimization problem using S-Procedure which enables us to obtain the globally optimal robust power control with fixed transmit beamforming. Further, we propose to find the optimal robust MISO beamforming via convex optimization and rank relaxation. A convergent iterative algorithm is presented to extend the robust solution for multiuser MIMO systems with both perfect and imperfect channel state information at the receiver (CSIR) to guarantee the worst-case SINR. Simulation results illustrate that the proposed joint robust power and beamforming optimization significantly outperforms the optimal robust power allocation with zeroforcing (ZF) beamformers, and more importantly enlarges the feasibility regions of a multiuser MIMO system.

Copyright (C) 2008 Gan Zheng et al. This is an open access article distributed under the Creative Commons Attribution License, which permits unrestricted use, distribution, and reproduction in any medium, provided the original work is properly cited.

\section{INTRODUCTION}

The rapid growth of wireless communications services has brought severe challenges to the design of reliable and efficient communications systems. In the future generation wireless systems, ubiquitous delivery of high-speed highquality services over air is anticipated whereas the physical susceptibility of a wireless channel such as fading continues to be a critical concern [1]. In response to this, multiantenna technologies, or widely known as multiple-input multipleoutput (MIMO) antenna systems, have emerged as an attractive means to provide diversity in the spatial domain without the need of bandwidth expansion and increase in transmit power. The amount of diversity benefit MIMO offers is directly linked to its enormous achievable capacity. It has been confirmed that not only can MIMO provide a substantial capacity gain to a single-user system (e.g., [2$8]$ ), such advantage is also even more apparent in multiuser systems [9-17]. With perfect channel state information (CSI), it is known that the channel capacity can be achieved using dirty-paper coding (DPC) in the MIMO downlink $[9,10]$. However, this nonlinear optimal strategy is not suitable for practical implementation and the beamforming alternatives have attracted much interests for their low complexity to realize the capacity enhancement [13-19].

While it is well known that CSI at the transmitter (CSIT) is not so important to achieve the capacity of an ergodic single-user MIMO channel at high signal-tonoise ratio (SNR) $[2,3,5]$, same is, however, not true for multiuser channels $[11,12]$. In a multiuser downlink system, for instance, the availability of CSIT would be essential to organize the users in a controlled way that the interference levels are kept minimal while enhancing the overall capacity by allowing users to transmit at the same time. Under the assumption with perfect CSIT and CSI at the receiver (CSIR), much have been understood so far. Unfortunately, this is never the case in practice, and channel error or uncertainty appears for many reasons. First, CSIT may be acquired through a quantized feedback channel from the receiver and there will be quantization errors in the CSIT. 
Channel estimation fidelity is also limited by the SNR at the estimation samples. In addition, a channel varies in time due to Doppler spread, which will cause more errors on the estimated CSIT or CSIR as time goes. There is no doubt that with channel uncertainty the achievable system capacity will go down (e.g., [20-23]) but more concernedly, for a system where users are required to achieve certain quality-ofservice (QoS) such as signal-to-interference-plus-noise ratio (SINR), the users' requirements are likely to be violated by a design based on the imperfect, and hence incorrect, CSIT/CSIR. Motivated by this reason, some recent studies aimed for robust beamforming designs to CSI uncertainty [14, 20-33].

In general, there are two ways to obtain a robust solution. One popular way is to examine the worst-case scenario and design the system under the worst-case channel condition $[14,24]$. Ideally, if the problem is indeed feasible and such design is obtained, it will ensure the users' requirements to be met for all possible channel error conditions. Alternatively, robustness could be obtained by a stochastic approach which takes a statistical viewpoint of the design problem and provides the needed robustness in the probabilistic sense [25]. Both the worst-case and the stochastic approaches have pros and cons against each other. Nevertheless, to get absolute robustness (i.e., performance guaranteed with probability one), worst-case designs are necessary and for this reason, this paper will investigate the worst-case approach to the robust beamforming design of a multiuser MIMO antenna system in the downlink, in the presence of both CSIT and CSIR uncertainties.

Some very recent robust techniques are reviewed as follows. The robust transceiver design for a single-user multicarrier MIMO system with various channel uncertainties was presented in [26]. In [27], a robust maximin approach was devised for a single-user MIMO system based on convex optimization. In [28], the robust transmit strategy to maximize the compound capacity, defined as the capacity of the worst-case realization within the uncertainty set, in single and multiuser rank-one Ricean MIMO channels was analyzed (see also [29]). It was also shown that beamforming is optimal for both single-user and multiuser settings. Robust adaptive beamforming using second-order cone program (SOCP) was proposed in [30] to deal with an arbitrary unknown signal steering vector mismatch based on the worst-case performance. For a multiuser MISO system (i.e., multiuser MIMO with single-antenna receivers) with individual QoS constraints, the robust beamforming vectors under the worst-case criteria were determined in $[14,31]$ given an imperfect channel covariance matrix. [32, 33] considered errors in the CSI matrices and studied the optimal power allocation with fixed beamforming vectors, again in a downlink multiuser MISO system. Most recently, some conservative design approaches that yield convex restrictions of the original robust design problem with imperfect CSIT and perfect CSIR were proposed in [34, 35]. This contemporary list of references indicates that despite the need to have a universal robust solution, how to ensure the worst-case QoS constraints of a multiuser MIMO system in the presence of CSI uncertainty is largely unknown.
This paper aims to devise a robust multiuser MIMO power and beamforming solution which optimizes the power allocation and the transmit and receives beamforming vectors of the users jointly, to minimize the overall transmit power in the downlink while guaranteeing the users' individual SINR constraints for every possible channel error conditions (i.e., a worst-case approach), in the presence of imperfect CSIT and perfect/imperfect CSIR uncertainty modeled by an ellipsoid. The motivation behind an ellipsoidal model is that it bounds the CSI errors to make possible such a worst-case design. In practice, CSI is measured in minimizing the mean-square-errors (MSE) and the CSI errors tend to be Gaussian. Such ellipsoidal bounding is thus appropriate and achievable with a small controllable outage probability. Previous works based on spherical or ellipsoidal CSI uncertainty regions can be found in $[23,27,28]$.

The technical difficulty of the design lies in the fact that the users' worst-case SINRs are hardly derivable without knowing the beamforming solution; yet, getting a loose bound on the SINR for robustness may result in huge transmit power penalty and worst, suffer a higher likelihood of the system becoming infeasible. In particular, this paper makes the following contributions.

(i) The optimal robust power allocation with fixed beamforming vectors (power-only optimal solution) is found via convex optimization.

(ii) A reformulation of the robust design using SProcedure [36] is presented for a multiuser MISO antenna system, which makes it possible to obtain the globally optimal robust solution via convex optimization and rank relaxation [37] with high probability (but not with probability one). More importantly, the proposed scheme results in a larger feasibility region than power-only optimization, where feasibility is declared if and only if there exist a power vector and transmit and receive beamforming vectors such that the worst-case SINR requirements are satisfied. This demonstrates that a joint optimization of the power allocation and the beamforming vectors is vital.

(iii) A convergent iterative algorithm is proposed to extend the robust multiuser MISO solution to a multiuser MIMO antenna system both with perfect and imperfect CSIR. Although not optimal, this algorithm guarantees the worst-case SINR at the mobile users. Simulation results will show that a significant reduction in transmit power is possible by using the proposed algorithm as compared to poweronly optimization methods.

The remainder of this paper is structured as follows. Section 2 introduces the system model for a multiuser MIMO with channel uncertainty and then formulates the robust optimization problem. In Section 3, we look at the robust design of a multiuser MISO antenna system first using an SINR bounding approach and then S-procedure. We will discuss how the optimal robust solution can be obtained using convex optimization and rank relaxation. Section 4 extends our results to a multiuser MIMO system and an 
iterative algorithm to jointly optimize the power allocation and the transmit and receive beamforming vectors for the users is presented. Simulation results will be presented in Section 5 and finally, we conclude this paper in Section 6.

Throughout this paper, complex scalar is represented by a lowercase letter and $|\cdot|$ denotes its modulus. $E[\cdot]$ denotes the mean of a random variable. Vectors and matrices are represented by bold lowercase and uppercase letters, respectively, and $\|\cdot\|$ is the Frobenius norm. The superscript $\dagger$ is used to denote the Hermitian transpose of a vector or matrix. $\mathbf{A} \otimes \mathbf{B}$ denotes the Kronecker product of matrices $\mathbf{A}$ and $\mathbf{B} . \mathbf{X} \succeq \mathbf{0}$ means that matrix $\mathbf{X}$ is positive semidefinite. eig $(\mathbf{X})$ returns the vector containing the eigenvalues of a square matrix $\mathbf{X}$ while trace $(\mathbf{A})$ denotes the trace of $\mathbf{A}$. $\operatorname{vec}(\mathbf{A})$ is a column vector by stacking all the elements of $\mathbf{A}$. Finally, $\mathbf{x} \sim \mathcal{C} \mathcal{N}(\mathbf{m}, \mathbf{V})$ denotes a vector of complex Gaussian entries with a mean vector of $\mathbf{m}$ and a covariance matrix of $\mathbf{V}$.

\section{SYSTEM MODEL AND PROBLEM FORMULATION}

\subsection{Multiuser MIMO in the downlink}

Consider an $M$-user MIMO antenna system where $n_{T}$ antennas are located at the base station and $n_{R}^{(m)}$ antennas are located at the $m$ th mobile station. Communication takes place in the downlink, that is, from the base station to the mobile receivers. As in [15-19], the system model is written as

$$
\tilde{\boldsymbol{s}}_{m}=\mathbf{r}_{m}^{\dagger}\left[\mathbf{H}_{m}\left(\sum_{n=1}^{M} \mathbf{t}_{n} \boldsymbol{s}_{n}\right)+\boldsymbol{\eta}_{m}\right], \quad m=1,2, \ldots, M,
$$

where

(i) $s_{m}$ is the digital symbol sent from user $m$ (complex scalar) with $\mathrm{E}\left[\left|s_{m}\right|^{2}\right]=1$;

(ii) $\tilde{s}_{m}$ is the estimated symbol at the mobile user $m$ (complex scalar);

(iii) $\mathbf{t}_{m}$ is the transmit beamforming vector for user $m$ $\left(n_{T} \times 1\right.$ complex vector $)$;

(iv) $\mathbf{r}_{m}$ is the receive beamforming vector for user $m$ $\left(n_{R}^{(m)} \times 1\right.$ complex vector) with $\left\|\mathbf{r}_{m}\right\|=1 ;$

(v) $\mathbf{H}_{m}$ is the MIMO channel from the transmitter to user $m\left(n_{R}^{(m)} \times n_{T}\right.$ complex matrix $) ;$

(vi) $\boldsymbol{\eta}_{m}$ is the noise vector $\sim \mathcal{C} \mathcal{N}\left(\mathbf{0}, N_{0} \mathbf{I}\right)$ at user $m\left(n_{R}^{(m)} \times\right.$ 1 complex vector).

The time index in the above model is omitted for convenience.

The SINR at the $m$ th user can be expressed as

$$
\Gamma_{m}=\frac{\left|\mathbf{r}_{m}^{\dagger} \mathbf{H}_{m} \mathbf{t}_{m}\right|^{2}}{\sum_{n=1, n \neq m}^{M}\left|\mathbf{r}_{m}^{\dagger} \mathbf{H}_{m} \mathbf{t}_{n}\right|^{2}+N_{0}},
$$

and the amount of power transmitted to this user is given by $\left\|\mathbf{t}_{m}\right\|^{2}$. The total transmit power of the base station is therefore

$$
P=\sum_{m=1}^{M}\left\|\mathbf{t}_{m}\right\|^{2}
$$

With perfect CSIT and CSIR, one would like to minimize the transmission cost for maintaining the users' QoS. Mathematically, this may be achieved by minimizing the overall transmit power with users' individual SINR constraints $\left\{\gamma_{m}\right\}$, that is,

$$
\mathbb{P}: \min _{\left\{\mathbf{t}_{m}, \mathbf{r}_{m}\right\}_{m=1}^{M}} P \quad \text { s.t. } \Gamma_{m} \geq \gamma_{m} \forall m .
$$

This problem has been extensively studied (e.g., [13-17]) although the globally optimal solution for a MIMO antenna system is still unknown.

With MIMO, spatial multiplexing (i.e., transmitting parallel substreams per user in the spatial domain) can be used to increase both the per-user and system capacity, but this is not considered here for simplicity. This restriction is also motivated by the fact that in many situations, singlestream transmission in multiuser MIMO is nearly optimal $[28,38-41]$.

\subsection{The definition of CSI and the ellipsoidal uncertainty region}

In this paper, CSIT and CSIR are estimated in two training periods. During the first one, CSIT, defined as the information about the channel matrices $\left\{\mathbf{H}_{m}\right\}$, may be estimated directly at the base station in the uplink. In particular, we model the imperfection of CSIT as an additive noisy matrix

$$
\mathbf{H}_{m}=\tilde{\mathbf{H}}_{\mathrm{T}}^{(m)}+\Delta \mathbf{H}_{m},
$$

where $\mathbf{H}_{m}$ is the actual channel matrix, $\tilde{\mathbf{H}}_{\mathbf{T}}^{(m)}$ denotes the CSIT estimates known to the base station, and $\Delta \mathbf{H}_{m}$ represents the CSIT uncertainty, bounded by the region

$$
\mathcal{U}_{\mathrm{T}}^{(m)}=\left\{\Delta \mathbf{H}_{m} \mid \operatorname{trace}\left(\Delta \mathbf{H}_{m} \mathbf{U}_{\mathrm{T}}^{(m)} \Delta \mathbf{H}_{m}^{\dagger}\right) \leq \xi_{\mathrm{T}}^{(m)^{2}}\right\},
$$

where $\mathbf{U}_{\top}^{(m)} \geq \mathbf{0}$ is a given matrix determined the orientation of the region and the parameter $\xi_{\mathrm{T}}^{(m)}$ controls the size of the region. (In practice, depending upon how the CSI is estimated (e.g., the length of the training sequence and the training power), the minimum MSE (MMSE) in the channel estimate will shed light on the required size of the region. ) In this paper, we will assume that $\mathbf{U}_{\top}^{(m)}$ is of full rank so that $U_{\top}^{(m)}$ has a geometric meaning of being an ellipsoid. It is said in [35] that such model may well be useful to characterize the quantization error in CSIT. In the rest of the paper, the knowledge for both $\left\{\tilde{\mathbf{H}}_{\mathrm{T}}^{(m)}\right\}$ and $\left\{\boldsymbol{U}_{\mathrm{T}}^{(m)}\right\}$ is assumed at the base station, based on which the robust transmit beamforming vectors $\left\{\mathbf{t}_{m}\right\}_{\forall m}$ are designed.

At the $m$ th mobile station, we define CSIR as the local information about the matrix or the vectors

$$
\mathbf{H}_{\mathrm{BF}}^{(m)} \triangleq \mathbf{H}_{m}\left[\begin{array}{llll}
\mathbf{t}_{1} & \mathbf{t}_{2} & \cdots & \mathbf{t}_{M}
\end{array}\right]
$$

which are the resultant channels after multiuser transmit beamforming. During the second training period, it can 
be estimated once the transmit beamforming design is completed. We find this CSIR definition necessary because the receive beamforming vector should be designed in accordance with the transmitted channels to maintain the required SINR. The matrix (7) can be estimated locally from the reception of the beamformed training sequences transmitted from the base station. The CSIR uncertainty can be modeled in the same way as for CSIT (8) so that

$$
\mathbf{H}_{\mathrm{BF}}^{(m)}=\widetilde{\mathbf{H}}_{\mathrm{BF}}^{(m)}+\Delta \mathbf{H}_{\mathrm{BF}}^{(m)},
$$

consists of an estimate $\tilde{\mathbf{H}}_{\mathrm{BF}}^{(m)}$ and the CSIR error $\Delta \mathbf{H}_{\mathrm{BF}}^{(m)}$, which is bounded by the region

$$
\mathcal{U}_{\mathrm{R}}^{(m)}=\left\{\Delta \mathbf{H}_{\mathrm{BF}}^{(m)} \mid \operatorname{trace}\left(\Delta \mathbf{H}_{\mathrm{BF}}^{(m)} \mathbf{U}_{\mathrm{R}}^{(m)} \Delta \mathbf{H}_{\mathrm{BF}}^{(m)}\right) \leq \xi_{\mathrm{R}}^{(m)^{2}}\right\}
$$

with the parameters $\mathbf{U}_{\mathbf{R}}^{(m)}(\succeq \mathbf{0})$ and $\xi_{\mathbf{R}}^{(m)}$. It is assumed that the mobile user $m$ has the knowledge of $\tilde{\mathbf{H}}_{\mathrm{BF}}^{(m)}$ and $\boldsymbol{U}_{\mathrm{R}}^{(m)}$, which is used for the design of the receive beamforming vector $\mathbf{r}_{m}$.

The generality of this model embraces the following situations as special cases, for example,

(a) no CSIT and perfect CSIR: $\widetilde{\mathbf{H}}_{\mathrm{T}}^{(m)} \rightarrow 0$ and $\widetilde{\mathbf{H}}_{\mathrm{BF}}^{(m)}=\mathbf{H}_{\mathrm{BF}}^{(m)}$ with $\xi_{\mathrm{R}}^{(m)} \rightarrow 0$;

(b) perfect CSIT and perfect CSIR: $\tilde{\mathbf{H}}_{\mathrm{T}}^{(m)}=\mathbf{H}_{m}$ with $\xi_{\mathrm{T}}^{(m)} \rightarrow 0$ and $\widetilde{\mathbf{H}}_{\mathrm{BF}}^{(m)}=\mathbf{H}_{\mathrm{BF}}^{(m)}$ with $\xi_{\mathrm{R}}^{(m)} \rightarrow 0 ;$

(c) imperfect CSIT and perfect CSIR: $\tilde{\mathbf{H}}_{\mathrm{T}}^{(m)} \neq \mathbf{0}$ with $\xi_{\mathrm{T}}^{(m)}>0$ and $\tilde{\mathbf{H}}_{\mathrm{BF}}^{(m)}=\mathbf{H}_{\mathrm{BF}}^{(m)}$ with $\xi_{\mathrm{R}}^{(m)} \rightarrow 0 ;$

(d) imperfect CSIT and imperfect CSIR: $\widetilde{\mathbf{H}}_{\mathrm{T}}^{(m)}, \widetilde{\mathbf{H}}_{\mathrm{BF}}^{(m)} \neq \mathbf{0}$ and $\xi_{\mathrm{T}}^{(m)}, \xi_{\mathrm{R}}^{(m)}>0$.

The foci of this paper are on cases (c) and (d) where the CSI errors are considered. In particular, for MISO systems to be discussed in Section 3, (c) will be studied. While for MIMO, both (c) and (d) are investigated (see Sections $4.1 \& 4.2$ for MIMO in (c) and Section 4.3 for MIMO in (d)).

One final point on the uncertainty model worth mentioning is that as a worst-case approach is adopted in this paper, the explicit statistical distribution of how the CSI error varies within the region is not important and therefore not exploited as usual in the worst-case optimization (as opposed to the stochastic optimization which takes into account the distribution of the error). It is, however, known that for MMSE channel estimation, $\Delta \mathbf{H}$ will tend to be Gaussian distributed, which we will assume in the simulation results section. The above ellipsoidal model, which has already been used in $[23,27,28,35]$, can be viewed as a deterministic modeling or simplification of the more sophisticated stochastic CSI uncertainty model.

\subsection{The robust optimization problem}

This paper adopts a worst-case methodology, whose solution is robust to every possible CSI error condition for a given $\left\{\tilde{\mathbf{H}}_{\mathbf{T}}^{(m)}, \tilde{\mathbf{H}}_{\mathbf{B F}}^{(m)}, \boldsymbol{U}_{\boldsymbol{\top}}^{(m)}, \boldsymbol{U}_{\mathbf{R}}^{(m)}\right\}$. In particular, our aim is to minimize the overall transmit power for ensuring the users'
SINR constraints by jointly optimizing the power allocation and the transmit-receive beamforming vectors of the users, with the aid of CSIT and CSIR, that is,

$$
\widetilde{\mathbb{P}}: \min _{\left\{\mathbf{t}_{m}, \mathbf{r}_{m}\right\}_{m=1}^{M}} P \quad \text { s.t. } \min _{\substack{\Delta \mathbf{H}_{m} \in \mathcal{U}_{\mathrm{T}}^{(m)} \\ \Delta \mathbf{H}_{\mathrm{BF}}^{(m)} \in \mathcal{U}_{\mathrm{R}}^{(m)}}} \Gamma_{m} \geq \gamma_{m} \forall m .
$$

Note that $\min \Gamma_{m}$ corresponds to the worst-case SINR for user $m$ given the CSI error regions. By ensuring $\min \Gamma_{m} \geq \gamma_{m}$, QoS assurance can be guaranteed for every possible CSI error condition.

\section{ROBUST MULTIUSER MISO}

In this section, we consider a MISO system where each receiver has only one antenna, and address the problem (10) with imperfect CSIT but perfect CSIR.

\subsection{The optimization}

The technical difficulty of solving (10) is obvious and even for a multiuser MISO setting, there has been no known optimal robust solution so far. In this section, to gain more insights and a deeper understanding of (10), we will look at a multiuser MISO antenna system where each mobile user has a single receive antenna (or $\mathbf{r}_{m}$ becomes a scalar). To distinguish the channel dimension from the MIMO case, we will use lowercase $\mathbf{h}$ to denote the respective channel vectors. The subscript $\mathrm{T}$ will be omitted for notational convenience as long as imperfect CSIR is not considered.

A simple observation shows that for MISO, the constraints in (10) can be rewritten as

$$
\widetilde{\mathbb{P}}_{\text {MISO }}: \min _{\left\{\mathbf{t}_{m}\right\}_{m=1}^{M}} P \text { s.t. } \min _{\Delta \mathbf{h}_{m} \in \mathcal{U}_{\mathrm{T}}^{(m)}} f_{m}\left(\Delta \mathbf{h}_{m}\right) \geq 0 \forall m,
$$

where

$$
\begin{gathered}
f_{m}\left(\Delta \mathbf{h}_{m}\right)=\left(\tilde{\mathbf{h}}_{m}+\Delta \mathbf{h}_{m}\right) \mathbf{Q}_{m}\left(\tilde{\mathbf{h}}_{m}^{\dagger}+\Delta \mathbf{h}_{m}^{\dagger}\right)-\gamma_{m} N_{0}, \\
\mathbf{Q}_{m} \triangleq \mathbf{t}_{m} \mathbf{t}_{m}^{\dagger}-\gamma_{m} \sum_{\substack{n=1 \\
n \neq m}}^{M} \mathbf{t}_{n} \mathbf{t}_{n}^{\dagger} .
\end{gathered}
$$

Problem (11) is actually a robust second-order cone programming (SOCP) problem in $\left\{\mathbf{t}_{m}\right\}$, and the constraints in (11) can be equivalently expressed as

$$
g\left(\Delta \mathbf{h}_{m},\left\{\mathbf{t}_{m}\right\}\right) \triangleq \sqrt{\gamma_{m}}\left\|\left[\begin{array}{c}
\left(\tilde{\mathbf{h}}_{m}+\Delta \mathbf{h}_{m}\right) \mathbf{t}_{1} \\
\left(\tilde{\mathbf{h}}_{m}+\Delta \mathbf{h}_{m}\right) \mathbf{t}_{2} \\
\vdots \\
\left(\tilde{\mathbf{h}}_{m}+\Delta \mathbf{h}_{m}\right) \mathbf{t}_{M} \\
\sqrt{N_{0}}
\end{array}\right]\right\|-\operatorname{Re}\left[\left(\tilde{\mathbf{h}}_{m}+\Delta \mathbf{h}_{m}\right) \mathbf{t}_{m}\right],
$$

According to [42], the SOCP constraints in (14) are not known to be tractable. A possible remedy is to derive a lower 
bound for the worst-case constraint for any $\Delta \mathbf{h}_{m} \in \mathcal{U}_{\boldsymbol{\top}}^{(m)}$. For the special case $\mathbf{U}_{\boldsymbol{T}}^{(m)}=\mathbf{I}$, this is possible and we describe this in the next subsection.

\subsection{Design by lower bounding the SINR}

To get around the difficulty of solving (11) with unknown $\left\{\Delta \mathbf{h}_{m}\right\}$, a simpler robust solution based on lower bounding the constraints is possible when $\mathbf{U}_{\mathrm{T}}^{(m)}=\mathbf{I}$ for all $m$. Using [26, Lemma 7.1], a lower bound for $f_{m}\left(\Delta \mathbf{h}_{m}\right)$, denoted by $f_{m}$, can be found as

$$
\begin{aligned}
\underline{f_{m}}= & -\gamma_{m}\left(\xi_{\mathrm{T}}^{(m)^{2}}+2 \xi_{\mathrm{T}}^{(m)} \rho\left(\tilde{\mathbf{h}}_{m}\right)\right) \sum_{n \neq m}\left\|\mathbf{t}_{n}\right\|^{2} \\
& -2 \xi_{\top}^{(m)} \rho\left(\tilde{\mathbf{h}}_{m}\right)\left\|\mathbf{t}_{m}\right\|^{2}+\tilde{\mathbf{h}}_{m} \mathbf{Q}_{m} \tilde{\mathbf{h}}_{m}^{\dagger}-\gamma_{m} N_{0} .
\end{aligned}
$$

The worst-case SINR can then be guaranteed by imposing $f_{m} \geq 0$. As a consequence, (11) can be suboptimally solved by

$$
\begin{aligned}
& \widetilde{\mathbb{Q}}_{\text {MISO }}: \min _{\left\{\mathbf{t}_{m}\right\}_{m=1}^{M}} P, \\
& \text { s.t. } \frac{\left|\tilde{\mathbf{h}}_{m} \mathbf{t}_{m}\right|^{2}-2 \xi_{\mathrm{T}}^{(m)} \rho\left(\tilde{\mathbf{h}}_{m}\right)\left\|\mathbf{t}_{m}\right\|^{2}}{\sum_{n=1, n \neq m}^{M}\left|\tilde{\mathbf{h}}_{m} \mathbf{t}_{n}\right|^{2}+Z+N_{0}} \geq \gamma_{m} \forall m,
\end{aligned}
$$

where $Z$ denotes $\left(\xi_{\mathrm{T}}^{(m)^{2}}+2 \xi_{\mathrm{T}}^{(m)} \rho\left(\tilde{\mathbf{h}}_{m}\right)\right) \sum_{n=1, n \neq m}^{M}\left\|\mathbf{t}_{n}\right\|^{2}$.

This problem is similar to that with perfect CSIT and there are algorithms (e.g., [17]) available to achieve the optimum. As will be shown later in the simulation results, however, the main drawback of this method is that the bound $f_{m}\left(\Delta \mathbf{h}_{m}\right)$ is too loose, which results in severe power penalty and even worse and diminishes the feasible region considerably. In the following subsection, we will show that the optimal solution of (11) could in fact be found without relying on SINR bounds.

\subsection{Optimal robust solution}

\subsubsection{S-procedure and convex optimization for power-only control}

The inferior performance of the design described above in Section 3.2 is because the bound is very loose and rarely achievable in most cases. In general, one can obtain robustness by the power-only optimization with fixed transmit beamforming vectors in (11). In [32], the optimal power allocation is found under several types of channel uncertainties including the ellipsoidal region considered in this paper. The main difference is that the work in [32] assumed that both the transmitter and the receiver share the same uncertainty region with a common channel estimate, which is hardly justifiable in practice. Secondly, the model in [32] also disallows their solution to deal with the case of imperfect CSIT and perfect CSIR, as we do in here. Now, we assume a fixed set of transmit beamforming vectors and find the optimal solution to the power control. The main result is based on S-procedure and given in Theorem 1 as follows.
Theorem 1. The optimal power control for the original beamforming problem (11) with fixed transmit beamforming vectors is given by the solution to the following semidefinite programming $(S D P)$ :

$$
\begin{aligned}
& \min _{\substack{\left\{p_{m}, s_{T}^{(m)} \geq 0\right\}_{m=1}^{M} \\
\text { s.t. }}}\left\{\begin{array}{cc}
{\left[\begin{array}{cc}
\tilde{\mathbf{h}}_{m} \mathbf{Q}_{m} \tilde{\mathbf{h}}_{m}^{\dagger}-\gamma_{m} N_{0}-s_{T}^{(m)} \xi_{T}^{(m)^{2}} & \tilde{\mathbf{h}}_{m} \mathbf{Q}_{m} \\
\mathbf{Q}_{m} \tilde{\mathbf{h}}_{m}^{\dagger} & \mathbf{Q}_{m}+s_{T}^{(m)} \mathbf{U}_{T}^{(m)}
\end{array}\right] \succeq \mathbf{0},} \\
\mathbf{Q}_{m}=p_{m} \mathbf{w}_{m} \mathbf{w}_{m}^{\dagger}-\gamma_{m} \sum_{\substack{n=1 \\
n \neq m}}^{M} p_{n} \mathbf{w}_{n} \mathbf{w}_{n}^{\dagger} \forall m,
\end{array}\right.
\end{aligned}
$$

where $\mathbf{w}_{m}$ denotes a fixed unit-norm transmit beamforming vector and the transmit beamforming vectors are given by $\mathbf{t}_{m}=$ $\sqrt{p_{m}} \mathbf{w}_{m}$.

Proof. Note in (11)-(13) that $\mathbf{Q}_{m}$ may, in general, be indefinite and it is possible that $f_{m}\left(\Delta \mathbf{h}_{m}\right)$ is not convex. However, according to S-lemma $[36,43]$, the constraint in (11), which is

$$
\begin{aligned}
& f_{m}\left(\Delta \mathbf{h}_{m}\right) \\
& =\left(\tilde{\mathbf{h}}_{m}+\Delta \mathbf{h}_{m}\right) \mathbf{Q}_{m}\left(\tilde{\mathbf{h}}_{m}^{\dagger}+\Delta \mathbf{h}_{m}^{\dagger}\right)-\gamma_{m} N_{0} \geq 0, \quad \forall \Delta \mathbf{h}_{m} \in \mathcal{U}_{\mathbf{T}}^{(m)},
\end{aligned}
$$

is equivalent to

$$
\begin{array}{rr}
{\left[\begin{array}{cc}
\tilde{\mathbf{h}}_{m} \mathbf{Q}_{m} \tilde{\mathbf{h}}_{m}^{\dagger}-\gamma_{m} N_{0}-s_{\mathrm{T}}^{(m)} \xi_{\mathrm{T}}^{(m)^{2}} & \tilde{\mathbf{h}}_{m} \mathbf{Q}_{m} \\
\mathbf{Q}_{m} \tilde{\mathbf{h}}_{m}^{\dagger} & \mathbf{Q}_{m}+s_{\mathrm{T}}^{(m)} \mathbf{U}_{\mathrm{T}}^{(m)}
\end{array}\right]} & \geq \mathbf{0}, \\
\exists s_{\mathrm{T}}^{(m)} & \geq 0 .
\end{array}
$$

With this equivalent constraint, we no longer need to derive the analytical form of the worst-case SINR or the worst-case $f_{m}\left(\Delta \mathbf{h}_{m}\right)$. As long as (21) is met, the constraint is guaranteed.

An interesting and useful fact about (21) is that $\Delta \mathbf{h}_{m}$ is not involved whereas the uncertainty structure is dealt with by the parameters, $\mathbf{U}_{\mathrm{T}}^{(m)}$ and $\xi_{\mathrm{T}}^{(m)}$.

\subsubsection{Joint power control and transmit beamforming design}

There are two main drawbacks of the power only optimization above in Section 3.3.1. Firstly, there is a power penalty caused by not allowing the optimization to be done jointly with the power allocation and the beamforming vectors. It will be shown in the simulation section that for MISO systems, the gap is negligible but for MIMO systems, the gap can be very significant (can be as large as $8 \mathrm{~dB}$ ), and the degradation grows with the number of users and the channel error bound $\xi$. Secondly and worst of all, the feasibility region of the joint power and beamforming design 
problem (22) tends to encompass that of (19) and this will have a detrimental implication on the likelihood of outage occurrence.

Although it is difficult to find an equivalent convex problem, if the power allocation and the transmit beamforming vectors of a MISO system are to be optimized jointly, in the following, we are about to show that it is possible to bound the problem (11) by a convex counterpart after rank relaxation. (We observe from the numerical results that the rank relaxation appears to be exact with high probability, allowing the globally optimal robust solution to be found via convex optimization, although analytical evidence is unavailable.) The main result is summarized in Theorem 2 below.

Theorem 2. The original robust problem (11) is relaxed as the following SDP problem:

$$
\begin{aligned}
& \min _{\left\{\mathbf{T}_{m} \succeq \mathbf{0}\right\}_{m=1}^{M}} \sum_{m=1}^{M}\{\text { trace }\}\left(\mathbf{T}_{m}\right), \\
& \left\{s_{T}^{(m)} \geq 0\right\}_{m=1}^{M} \\
& \text { s.t. }\left[\begin{array}{cc}
\tilde{\mathbf{h}}_{m} \mathbf{Q}_{m} \tilde{\mathbf{h}}_{m}^{\dagger}-\gamma_{m} N_{0}-s_{T}^{(m)} \xi_{T}^{(m)^{2}} & \tilde{\mathbf{h}}_{m} \mathbf{Q}_{m} \\
\mathbf{Q}_{m} \tilde{\mathbf{h}}_{m}^{\dagger} & \mathbf{Q}_{m}+\boldsymbol{s}_{T}^{(m)} \mathbf{U}_{T}^{(m)}
\end{array}\right] \succeq \mathbf{0} \quad \forall \mathrm{m},
\end{aligned}
$$

where

$$
\mathbf{Q}_{m} \triangleq \mathbf{T}_{m}-\gamma_{m} \sum_{\substack{n=1 \\ n \neq m}}^{M} \mathbf{T}_{n} \quad \forall m .
$$

The problem (22) is convex and hence can be optimally solved.

Proof. The proof about the equivalent constraint in (22) is the same to that in Theorem 1. Using (21), and introducing the transmit covariance matrices $\left\{\mathbf{T}_{m} \triangleq \mathbf{t}_{m} \mathbf{t}_{m}^{\dagger} \succeq \mathbf{0}\right\}$, (11) becomes

$$
\min _{\substack{\left\{\mathbf{T}_{m} \geq \mathbf{0}\right\}_{m=1}^{M} \\\left\{s_{\mathrm{T}}^{(m)} \geq 0\right\}_{m=1}^{M}}} \sum_{m=1}^{M} \operatorname{trace}\left(\mathbf{T}_{m}\right)
$$

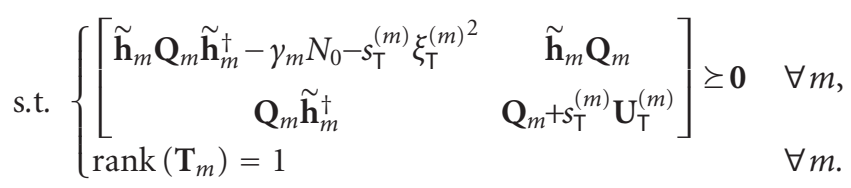

Apparently, (24) (and hence (11)) is the same as (22) except that the rank-1 constraints are missing in (22). Due to this rank-relaxation, in general, (22) gives a lower bound for the problem (24). As a result, the original problem (11) is lower bounded by (22).

The advantage of (22) is substantial because it is an SDP problem and hence can be optimally solved efficiently. Moreover, we observe from the simulation results that in most cases (22) gives rank-1 solutions if all $\left\{\mathbf{U}_{\mathrm{T}}^{(m)}\right\}$ are of fullrank (i.e., $\boldsymbol{U}_{\mathrm{T}}^{(m)}$ are indeed ellipsoids), which means that the relaxation is exact and the optimal robust solution to (11) can thus be found from solving (22). If the SDP does not offer a rank-1 solution, then a countermeasure is needed (see Section 3.3.4).

\subsubsection{Interpretation of (22) versus (11) with perfect CSIT}

At first, (22) may look quite different from (11) with perfect CSIT (or when $\Delta \mathbf{h}_{m}=\mathbf{0}$ ), and the original SINR constraints in (22) are not explicit. However, the two problems can be well linked with each other by their duals. In Appendix A, we show that the dual of (22) can be written as

$$
\begin{aligned}
& \max _{\left\{\lambda_{m}, \mathbf{V}_{m}, \mathbf{v}_{m}\right\}_{m=1}^{M}} \sum_{m=1}^{M} \lambda_{m} \gamma_{m} N_{0}, \\
& \text { s.t. }\left\{\begin{array}{l}
\mathbf{I}-\lambda_{m}\left(\tilde{\mathbf{h}}_{m}^{\dagger} \tilde{\mathbf{h}}_{m}+\frac{\left.\mathbf{v}_{m} \tilde{\mathbf{h}}_{m}+\tilde{\mathbf{h}}_{m}^{\dagger} \mathbf{v}_{m}^{\dagger}+\mathbf{V}_{m}\right)}{\lambda_{m}} \tilde{c}_{\substack{n=1 \\
n \neq m}}^{M} \gamma_{n} \lambda_{n}\left(\tilde{\mathbf{h}}_{n}^{\dagger} \tilde{\mathbf{h}}_{n}+\frac{\mathbf{v}_{n} \tilde{\mathbf{h}}_{n}+\tilde{\mathbf{h}}_{n}^{\dagger} \mathbf{v}_{n}^{\dagger}+\mathbf{V}_{n}}{\lambda_{n}}\right) \geq \mathbf{0},\right. \\
\quad+\sum_{m} \frac{\operatorname{trace}\left(\mathbf{U}_{\top}^{(m)} \mathbf{V}_{m}\right)}{\lambda_{m}}, \\
{\left[\begin{array}{ll}
\lambda_{m} & \mathbf{v}_{m}^{\dagger} \\
\mathbf{v}_{m} & \mathbf{V}_{m}^{(m)^{2}}
\end{array}\right] \succeq \mathbf{0} \quad \forall m .}
\end{array}\right.
\end{aligned}
$$

On the other hand, the dual of (11) is given by [14]

$$
\begin{aligned}
& \max _{\left\{\lambda_{m} \geq 0\right\}_{m=1}^{M}} \sum_{m=1}^{M} \lambda_{m} \gamma_{m} N_{0}, \\
& \text { s.t. } \mathbf{I}-\lambda_{m} \mathbf{h}_{m}^{\dagger} \mathbf{h}_{m}+\sum_{\substack{n=1 \\
n \neq m}}^{M} \gamma_{n} \lambda_{n} \mathbf{h}_{n}^{\dagger} \mathbf{h}_{n} \succeq \mathbf{0} \forall m .
\end{aligned}
$$

Comparing (25) with (26), we can actually see that they are similar. In particular, the matrix

$$
\tilde{\mathbf{h}}_{m}^{\dagger} \tilde{\mathbf{h}}_{m}+\frac{\mathbf{v}_{m} \tilde{\mathbf{h}}_{m}+\tilde{\mathbf{h}}_{m}^{\dagger} \mathbf{v}_{m}^{\dagger}+\mathbf{V}_{m}}{\lambda_{m}}
$$

in (25) can be interpreted as the equivalent channel covariance matrix $\mathbf{h}_{m}^{\dagger} \mathbf{h}_{m}$ in (26). Nevertheless, (25) tends to require a larger objective value (i.e., $\sum_{m} \lambda_{m} \gamma_{m} N_{0}$ ) to respond to the channel uncertainty parameters (i.e., $\mathbf{U}_{\mathrm{T}}^{(m)}$ and $\xi_{\mathrm{T}}^{(m)}$ ), and this can be seen by the fact that the constraint of $\lambda_{m}$ in (25) is stricter than that in (26) because

$$
\lambda_{m} \geq \frac{\operatorname{trace}\left(\mathbf{U}_{\mathrm{T}}^{(m)} \mathbf{V}_{m}\right)}{\xi_{\mathrm{T}}^{(m)^{2}}} \geq 0 .
$$

\subsubsection{Feasibility, rank-1 solutions, and a countermeasure}

Thus far, little is understood about the feasibility of linear multiuser MIMO antenna systems with imperfect and even perfect CSIT. Despite the contributions in Section 3.2, the exact feasibility issue of a multiuser MIMO antenna system with imperfect CSIT is still not known. However, what we 
Example: Consider the system with the parameters

$$
\begin{aligned}
\widetilde{\mathbf{H}}_{1} & =[0.6607-0.4199 i, 0.8687-0.1855 i] \\
\widetilde{\mathbf{H}}_{2} & =[-0.1764+0.8788 i,-0.5003-1.0952 i], \\
\mathbf{U}_{T}^{(1)} & =\left[\begin{array}{cc}
0.4235 & -0.4528-0.1738 i \\
-0.4528+0.1738 i & 0.5946
\end{array}\right] \text { with eig }\left(\mathbf{U}_{T}^{(1)}\right)=\left[\begin{array}{l}
0.0166 \\
1.0015
\end{array}\right], \\
\mathbf{U}_{T}^{(2)} & =\left[\begin{array}{cc}
0.3646 & 1.2620+0.2997 i \\
1.2620-0.2997 i & 4.6347
\end{array}\right] \text { with eig }\left(\mathbf{U}_{T}^{(2)}\right)=\left[\begin{array}{l}
0.0014 \\
4.9978
\end{array}\right], \\
\gamma_{1} & =0.4174, \quad \gamma_{2}=1.3475, \quad \xi_{T}^{(1)}=\xi_{T}^{(2)}=0.1, \quad N_{0}=1 .
\end{aligned}
$$

Solving the SDP by the rank-relaxation method yields the following solution

$$
\begin{aligned}
\mathbf{T}_{1} & =\left[\begin{array}{cc}
1.2727 & 2.4240+0.8372 i \\
2.4240-0.8372 i & 5.1674
\end{array}\right] \quad \text { with eig }\left(\mathbf{T}_{1}\right)=\left[\begin{array}{c}
0 \\
6.4401
\end{array}\right], \\
\mathbf{T}_{2} & =\left[\begin{array}{cc}
1.8038 & 3.6163+1.2198 i \\
3.6163-1.2198 i & 8.2732
\end{array}\right] \quad \text { with eig }\left(\mathbf{T}_{2}\right)=\left[\begin{array}{c}
0.0356 \\
10.0414
\end{array}\right], \\
P & =\operatorname{trace}\left(\mathbf{T}_{1}+\mathbf{T}_{2}\right)=16.5172 .
\end{aligned}
$$

Using the method mentioned in Section 3.3.4 to solve (19), we can get

$$
\mathbf{t}_{1}=\left[\begin{array}{c}
-1.2158 \\
-2.3156+0.7997 i
\end{array}\right], \quad \mathbf{t}_{2}=\left[\begin{array}{c}
-1.5739 \\
-2.9976+1.0352 i
\end{array}\right], \quad P=\left\|\mathbf{t}_{1}\right\|^{2}+\left\|\mathbf{t}_{2}\right\|^{2}=20.0140
$$

FIgURE 1: A numerical example showing how the countermeasure works.

can say is that if (22) is infeasible, the original problem (11) cannot be feasible since (22) is a relaxed version. The existence of the proposed robust solution relies on whether the problem is feasible for a particular channel realization and error condition. If the problem happens to be infeasible, then an outage will be declared. In practice, it may mean that the users' requirements will have to be degraded or the transmission will have to be postponed until the channels improve to a better state.

In addition, even if (22) is feasible, it may return a solution with rank higher than 1 . Whether an all-rank-1 solution exists for (22) is not known. In this paper, if (22) gives higher-rank solutions, the following countermeasure, which optimizes only the power allocation of the users for a given set of fixed beamforming vectors in Section 3.3.1 will be in place.

In this case, $\mathbf{w}_{m}$ may be chosen as, for instance, the zeroforcing (ZF) beamforming vectors [16] or the principal eigenvector of the optimal $\mathbf{T}_{m}$ obtained from the SDP. The latter appears to be more useful because $\mathrm{ZF}$ vectors may not always exist. In some cases when an all-rank-1 solution to (22) is not available, the power-only optimization by choosing the dominant eigenvector as the beamforming vector will produce a contingent robust solution to (11). To illustrate how it works, a numerical example is given in Figure 1.

\section{EXTENSION TO MULTIUSER MIMO}

In this section, we extend our results to a multiuser MIMO antenna system in the downlink, and the joint optimization of the transmit and receive beamforming vectors is anticipated. Although a lower bounding approach, similar to Section 3.2, may be possible, the SINR bounds would be too loose to be useful. As such, we focus on how the SDP reformulation in Section 3.3 is extended to cope with the MIMO optimization. It is, however, well known that a joint optimization of transmit and receive beamforming vectors of a multiuser system is not convex. Even with perfect CSIT/CSIR, the optimal solution is not known, let alone with imperfect CSI. In the following, we first look at the case with imperfect CSIT and perfect CSIR as for the multiuser MISO case in Section 3. The case with imperfect CSIR will be addressed later in Section 4.3.

In the case of imperfect CSIT and perfect CSIR, the worst-case SINR is expressed as [15]

$$
\begin{aligned}
& \min _{\Delta \mathbf{H}_{m} \in \mathcal{U}_{\top}^{(m)}} \Gamma_{m} \\
& =\min _{\Delta \mathbf{H}_{m} \in \mathcal{U}_{\top}^{(m)}} \mathbf{t}_{m}^{\dagger} \mathbf{H}_{m}^{\dagger} \times\left[\sum_{\substack{n=1 \\
n \neq m}}^{M} \mathbf{H}_{m} \mathbf{t}_{n} \mathbf{t}_{n}^{\dagger} \mathbf{H}_{m}^{\dagger}+N_{0} \mathbf{I}\right] \mathbf{H}_{m} \mathbf{t}_{m},
\end{aligned}
$$

and is very difficult to evaluate. In the following, a suboptimal approach to promise the worst-case SINR will be presented. The base station assumes that the mobile user has the same knowledge of CSI. The transmit beamforming vectors $\left\{\mathbf{t}_{m}\right\}$ (also with the power allocation) and virtual receive beamforming vectors $\left\{\overline{\mathbf{r}}_{m}\right\}$ are optimized jointly at the base station based on the CSIT (i.e., $\left\{\tilde{\mathbf{H}}_{m}\right\}$ and $\mathcal{U}_{\mathrm{T}}^{(m)}$ ). After that the actual receive beamforming vectors $\left\{\mathbf{r}_{m}\right\}$ are optimized locally at the mobile receivers based on the perfect CSIR, that is, $\mathbf{H}_{\mathrm{BF}}^{(m)} \triangleq \mathbf{H}_{m}\left[\mathbf{t}_{1} \cdots \mathbf{t}_{M}\right]$. Note that $\left\{\overline{\mathbf{r}}_{m}\right\}$ are the only auxiliary variables to facilitate the design of $\left\{\mathbf{t}_{m}\right\}$. 
To obtain a robust solution of $\left\{\mathbf{t}_{m}\right\}$ to (10), an iterative optimization algorithm is proposed, which optimizes one set of variables at a time while keeping others fixed and iterates from one optimization to another to converge to the joint-optimized state, with the aid of CSIT (see Section 4.1). Then the corresponding solution of $\mathbf{r}_{m}$ is learnt locally at the $m$ th mobile receiver, based on the perfect CSIR. Because the mobile user actually has perfect CSIR, such a design results in a lower bound for the achievable worst-case SINR.

Similar to the MISO case, the constraints in (10) for the MIMO systems can be simplified as

$$
\begin{aligned}
& \min _{\Delta \mathbf{H}_{m} \in \mathcal{U}_{\mathrm{T}}^{(m)}} F_{m}\left(\Delta \mathbf{H}_{m}\right) \\
& \triangleq\left|\mathbf{r}_{m}^{\dagger} \mathbf{H}_{m} \mathbf{t}_{m}\right|^{2}-\gamma_{m}\left(\sum_{\substack{n=1 \\
n \neq m}}^{M}\left|\mathbf{r}_{m}^{\dagger} \mathbf{H}_{m} \mathbf{t}_{n}\right|^{2}+N_{0}\right) \geq 0 .
\end{aligned}
$$

\subsection{Optimization at the base station, $\left\{\mathbf{t}_{m}\right\}_{m=1}^{M}$}

\subsubsection{Transmit beamforming}

For a given set of the virtual receive beamforming vectors $\left\{\overline{\mathbf{R}}_{m} \triangleq \overline{\mathbf{r}}_{m} \overline{\mathbf{r}}_{m}^{\dagger}\right\}$, we consider how the transmit beamforming vectors $\left\{\mathbf{T}_{m}\right\}$ can be optimized by first rewriting (30) as

$$
\begin{aligned}
F_{m}\left(\Delta \mathbf{H}_{m}\right)= & \overline{\mathbf{r}}_{m}^{\dagger} \widetilde{\mathbf{H}}_{m} \mathbf{Q}_{m} \widetilde{\mathbf{H}}_{m}^{\dagger} \overline{\mathbf{r}}_{m}+\overline{\mathbf{r}}_{m}^{\dagger} \widetilde{\mathbf{H}}_{m} \mathbf{Q}_{m} \Delta \mathbf{H}_{m}^{\dagger} \overline{\mathbf{r}}_{m} \\
& +\overline{\mathbf{r}}_{m}^{\dagger} \Delta \mathbf{H}_{m} \mathbf{Q}_{m} \tilde{\mathbf{H}}_{m}^{\dagger} \overline{\mathbf{r}}_{m}+\overline{\mathbf{r}}_{m}^{\dagger} \Delta \mathbf{H}_{m} \mathbf{Q}_{m} \Delta \mathbf{H}_{m}^{\dagger} \overline{\mathbf{r}}_{m}-\gamma_{m} N_{0},
\end{aligned}
$$

where $\mathbf{Q}_{m}$ is defined in (13). This constraint can further be re-expressed using vector operation and Kronecker product as

$$
\begin{aligned}
F_{m}\left(\Delta \mathbf{H}_{m}\right)= & \operatorname{trace}\left(\Delta \mathbf{H}_{m} \mathbf{Q}_{m} \tilde{\mathbf{H}}_{m}^{\dagger} \overline{\mathbf{R}}_{m}\right)+\operatorname{trace}\left(\Delta \mathbf{H}_{m}^{\dagger} \overline{\mathbf{R}}_{m} \tilde{\mathbf{H}}_{m} \mathbf{Q}_{m}\right) \\
& +\operatorname{trace}\left(\Delta \mathbf{H}_{m}^{\dagger} \overline{\mathbf{R}}_{m} \Delta \mathbf{H}_{m} \mathbf{Q}_{m}\right)+\operatorname{trace}\left(\tilde{\mathbf{H}}_{m} \mathbf{Q}_{m} \tilde{\mathbf{H}}_{m}^{\dagger} \overline{\mathbf{R}}_{m}\right) \\
& -\gamma_{m} N_{0} \\
= & \operatorname{vec}\left(\Delta \mathbf{H}_{m}^{\dagger}\right)^{\dagger} \operatorname{vec}\left(\mathbf{Q}_{m} \widetilde{\mathbf{H}}_{m} \overline{\mathbf{R}}_{m}\right) \\
& +\operatorname{vec}\left(\mathbf{Q}_{m} \tilde{\mathbf{H}}_{m} \overline{\mathbf{R}}_{m}\right)^{\dagger} \operatorname{vec}\left(\Delta \mathbf{H}_{m}^{\dagger}\right) \\
& +\operatorname{vec}\left(\Delta \mathbf{H}_{m}^{\dagger}\right)^{\dagger}\left(\overline{\mathbf{R}}_{m} \otimes \mathbf{Q}_{m}\right) \operatorname{vec}\left(\Delta \mathbf{H}_{m}^{\dagger}\right) \\
& +\operatorname{trace}\left(\widetilde{\mathbf{H}}_{m} \mathbf{Q}_{m} \tilde{\mathbf{H}}_{m}^{\dagger} \overline{\mathbf{R}}_{m}\right)-\gamma_{m} N_{0},
\end{aligned}
$$

and $\Delta \mathbf{H}_{m} \in \mathcal{U}_{\mathrm{\top}}^{(m)}$ can be rewritten as

$$
\begin{aligned}
\operatorname{trace}\left(\Delta \mathbf{H}_{m} \mathbf{U}_{\mathrm{\top}}^{(m)} \Delta \mathbf{H}_{m}^{\dagger}\right) & =\operatorname{vec}\left(\Delta \mathbf{H}_{m}^{\dagger}\right)^{\dagger}\left(\mathbf{I} \otimes \mathbf{U}_{\mathrm{T}}^{(m)}\right) \operatorname{vec}\left(\Delta \mathbf{H}_{m}^{\dagger}\right) \\
& \leq \xi_{\mathrm{T}}^{(m)^{2}}
\end{aligned}
$$

Using the S-lemma with known $\left\{\overline{\mathbf{R}}_{m}\right\}$, (10) can be reformulated using rank relaxation as follows:

$$
\begin{aligned}
& \min _{\substack{\left\{\mathbf{T}_{m} \geq \mathbf{0}\right\}_{m=1}^{M} \\
\left\{s_{\mathrm{T}}^{(m)} \geq 0\right\}_{m=1}^{M}}} \sum_{m=1}^{M} \operatorname{trace}\left(\mathbf{T}_{m}\right), \\
& \text { s.t. }\left[\begin{array}{cc}
D & \operatorname{vec}\left(\mathbf{Q}_{m} \widetilde{\mathbf{H}}_{m} \mathbf{R}_{m}\right)^{\dagger} \\
\operatorname{vec}\left(\mathbf{Q}_{m} \widetilde{\mathbf{H}}_{m} \mathbf{R}_{m}\right) & \mathbf{R}_{m} \otimes \mathbf{Q}_{m}+s_{\mathrm{T}}^{(m)} \mathbf{I} \otimes \mathbf{U}_{\mathrm{T}}^{(m)}
\end{array}\right] \succeq \mathbf{0}, \\
& \forall m,
\end{aligned}
$$

where $D$ denotes trace $\left(\widetilde{\mathbf{H}}_{m} \mathbf{Q}_{m} \tilde{\mathbf{H}}_{m}^{\dagger} \mathbf{R}_{m}\right)-\gamma_{m} N_{0}-s_{\mathrm{T}}^{(m)} \xi_{\mathrm{T}}^{(m)^{2}}$.

Solving this convex SDP problem gives the optimal $\left\{\mathbf{T}_{m}\right\}$ for a given $\left\{\overline{\mathbf{R}}_{m}\right\}$. The dimension of the matrix $\mathbf{R}_{m} \otimes \mathbf{Q}_{m}$ in the constraint of (34) is $n_{T} n_{R}^{(m)} \times n_{T} n_{R}^{(m)}$. According to the analysis in [44, Chapter 6], the associated complexity to solve the SDP is $\mathcal{O}\left(\left(n_{T} \sum_{m=1}^{M} n_{R}^{(m)}\right)^{6.5}\right)$ per accuracy digit. It should be noted that as discussed earlier in Section 3.3.3, however, the rank-1 solution $\left\{\mathbf{t}_{m}\right\}$ may not be known but can be dealt with in the similar way.

\subsubsection{Virtual receive beamforming}

The optimization of the virtual receive beamforming vectors $\left\{\overline{\mathbf{r}}_{m}\right\}$ is also based on the CSIT. For a given user $m$, we propose to optimize the virtual receiver $\overline{\mathbf{r}}_{m}$ in order to maximize the worst-case $F_{m}\left(\Delta \mathbf{H}_{m}\right)$. In particular, $\overline{\mathbf{r}}_{m}$ is chosen to be the solution of the following problem

$$
\begin{aligned}
& \max _{\left\|\overline{\mathbf{r}}_{m}\right\|=1} \min _{\Delta \mathbf{H}_{m} \in \mathcal{U}_{\mathrm{\top}}^{(m)}} F_{m}\left(\Delta \mathbf{H}_{m},\left\{\mathbf{T}_{m}\right\}, \overline{\mathbf{r}}_{m}\right), \\
& \text { s.t. trace }\left(\Delta \mathbf{H}_{m} \mathbf{U}_{\mathrm{T}}^{(m)} \Delta \mathbf{H}_{m}^{\dagger}\right) \leq \xi_{\mathrm{T}}^{(m)^{2}},
\end{aligned}
$$

where $F_{m}(\cdot)$ in (32) is evaluated. It will be shown later in Section 4.1.3 that this optimization criterion enables the construction of a convergent iterative algorithm for the joint optimization of the transmit and receive beamforming vectors.

Once again, we find the S-lemma and rank relaxation very useful in transforming the problem into an SDP for ease of solving. Hence, (35) becomes

$$
\begin{array}{rl}
\max _{g, \overline{\mathbf{R}}_{m} \geq \mathbf{0},},\left\{s_{\mathrm{T}}^{(m)} \geq 0\right\}_{m=1}^{M} & g, \\
\text { s.t. } & \left\{\begin{array}{cc}
G & \operatorname{vec}\left(\mathbf{Q}_{m} \tilde{\mathbf{H}}_{m} \overline{\mathbf{R}}_{m}\right)^{\dagger} \\
& {\left[\begin{array}{cc}
\operatorname{vec}\left(\mathbf{Q}_{m} \tilde{\mathbf{H}}_{m} \overline{\mathbf{R}}_{m}\right) & \overline{\mathbf{R}}_{m} \otimes \mathbf{Q}_{m}+s_{\mathrm{T}}^{(m)} \mathbf{I} \otimes \mathbf{U}_{\mathrm{T}}^{(m)}
\end{array}\right] \geq \mathbf{0},} \\
\operatorname{trace}\left(\overline{\mathbf{R}}_{m}\right)=1 .
\end{array}\right.
\end{array}
$$

where $G$ denotes trace $\left(\widetilde{\mathbf{H}}_{m} \mathbf{Q}_{m} \widetilde{\mathbf{H}}_{m}^{\dagger} \overline{\mathbf{R}}_{m}\right)-\gamma_{m} N_{0}-s_{\mathrm{T}}^{(m)} \xi_{\mathrm{T}}^{(m)^{2}}-g$.

As the optimization of $\left\{\mathbf{T}_{m}\right\}$ requires only the knowledge of $\left\{\overline{\mathbf{R}}_{m}\right\}$, rather than $\left\{\overline{\mathbf{r}}_{m}\right\}$, whether or not (36) returns a rank-1 solution is unimportant since a rank-1 solution does always exist [45] and it only needs to be extracted after the iterative algorithm in the next subsection converges. 


\subsubsection{The iterative algorithm}

The above results can be iteratively combined to reach a joint optimization state so that $\left\{\mathbf{t}_{m}\right\}$ can be found. The proposed algorithm is outlined as follows. Note that we will use the notation $a^{[n]}$ to denote the optimizing variable $a$ at the $n$th iterate.

(1) Setting the iteration index $n=1$, initialize the receive covariance matrices $\left\{\overline{\mathbf{R}}_{m}^{[0]}\right\}=\left\{\overline{\mathbf{r}}_{m}^{[0]}\right\}\left\{\overline{\mathbf{r}}_{m}^{[0]}\right\}^{\dagger}$, where $\overline{\mathbf{r}}_{m}^{[0]}$ is chosen to match the principle left singular vector of channel $\tilde{\mathbf{H}}_{m}$.

(2) Solve (34) to obtain the corresponding optimal transmit covariance matrices $\left\{\mathbf{T}_{m}^{[n]}\right\}$.

(3) Solve (36) to obtain the corresponding optimal receive covariance matrices $\left\{\overline{\mathbf{R}}_{m}^{[n]}\right\}$.

(4) Update $n:=n+1$ and go back to step (2) until convergence.

The convergence of the above algorithm will be analyzed in the next subsection. At convergence, we will have the steady-state joint solution $\left\{\mathbf{T}_{m}^{[\infty]}, \overline{\mathbf{R}}_{m}^{[\infty]}\right\}$. If $\left\{\mathbf{T}_{m}^{[\infty]}\right\}$ are all of rank one, the robust transmit beamforming vectors $\left\{\mathbf{t}_{m}\right\}$ can be readily obtained from the Cholesky decomposition of $\left\{\mathbf{T}_{m}^{[\infty]}\right\}$. Otherwise, the technique described in Section 3.3.3 is needed to get a suboptimal solution for $\left\{\mathbf{t}_{m}\right\}$ for a given $\left\{\mathbf{T}_{m}^{[\infty]}, \overline{\mathbf{R}}_{m}^{[\infty]}\right\}$. However, due to the rank relaxation in the optimization of $\left\{\mathbf{t}_{m}\right\}$, it is possible that (10) is feasible but the above algorithm does not return a feasible rank-1 solution. How the actual receive beamforming vectors $\left\{\mathbf{r}_{m}\right\}$ are obtained will be addressed in Section 3.2.

\subsubsection{Convergence analysis}

Given a feasible initial point to start the iteration, we can prove that the proposed algorithm is convergent. Nevertheless, it is worth mentioning that as the problem is nonconvex, the proposed algorithm may converge only to the local optimum and the effect of the choice of the initial receive covariance matrices is still unknown. In the following, we start the proof by denoting the total transmit power at the $n$th iteration as $P^{[n]}$ and considering the $n$th and the $(n+1)$ th iterates.

Proof. At step (2) of the $n$th iteration, for a given set of $\left\{\overline{\mathbf{R}}_{m}^{[n]}\right\}_{m=1}^{M}$, the optimal $\left\{\mathbf{T}_{m}^{[n]}\right\}_{m=1}^{M}$ are obtained. Therefore, after that, we have a joint feasible solution

$$
\left\{\mathbf{T}_{m}^{[n]}, \overline{\mathbf{R}}_{m}^{[n]}\right\}_{m=1}^{M},
$$

which gives a sum-power of $P^{[n]}$ and $F_{m}\left(\Delta \mathbf{H}_{m}, \quad\left\{\mathbf{T}_{m}^{[n]}\right\}\right.$, $\left.\overline{\mathbf{R}}_{m}^{[n]}\right)=0$.

At step (3) of the $n$th iteration, since $\left\{\overline{\mathbf{R}}_{m}^{[n+1]}\right\}_{m=1}^{M}$ are updated for a given set of $\left\{\mathbf{T}_{m}^{[n]}\right\}_{m=1}^{M}$ to maximize $F_{m}\left(\Delta \mathbf{H}_{m},\left\{\mathbf{T}_{m}(n)\right\}, \overline{\mathbf{R}}_{m}\right)$, that is,

$$
\overline{\mathbf{R}}_{m}^{[n+1]}=\arg \max _{\overline{\mathbf{R}}_{m}} F_{m}\left(\Delta \mathbf{H}_{m},\left\{\mathbf{T}_{m}^{[n]}\right\}, \overline{\mathbf{R}}_{m}\right),
$$

we have

$$
\begin{aligned}
F_{m}\left(\Delta \mathbf{H}_{m},\left\{\mathbf{T}_{m}^{[n]}\right\}, \overline{\mathbf{R}}_{m}^{[n+1]}\right) & \geq F_{m}\left(\Delta \mathbf{H}_{m},\left\{\mathbf{T}_{m}^{[n]}\right\} \overline{\mathbf{R}}_{m}^{[n]}\right) \\
& =0,
\end{aligned}
$$

which means that the worst-case SINR requirements are over-satisfied with the same sum-power $P^{[n]}$ by the feasible solution

$$
\left\{\mathbf{T}_{m}^{[n]}, \overline{\mathbf{R}}_{m}^{[n+1]}\right\}_{m=1}^{M}
$$

At step (2) of the $(n+1)$ th iteration, we have another feasible solution

$$
\left\{\mathbf{T}_{m}^{[n+1]}, \overline{\mathbf{R}}_{m}^{[n+1]}\right\}_{m=1}^{M}
$$

with the sum-power of $P^{[n+1]}$. By definition, as $\left\{\mathbf{T}_{m}^{[n+1]}\right\}_{m=1}^{M}$ is obtained by minimizing the total transmit power with known $\left\{\overline{\mathbf{R}}_{m}^{[n+1]}\right\}_{m=1}^{M}$, then we have always $P^{[n]} \geq P^{[n+1]}$. As a result, the total power is monotonically decreasing (and obviously lower bounded by 0 ) and hence the proposed algorithm converges, which completes the proof.

\subsection{Optimization at the $m$ th mobile receiver, $\mathbf{r}_{m}$}

With the effective channels $\left\{\mathbf{H}_{m} \mathbf{t}_{n}\right\}$ being learnt perfectly at the mobile receiver, the corresponding optimal receive beamforming vectors $\left\{\mathbf{r}_{m}\right\}$ are well known to follow the MMSE criteria [46] and given by

$$
\mathbf{r}_{m}=\varsigma_{m}\left[\sum_{n=1}^{M} \mathbf{H}_{m} \mathbf{t}_{n}\left(\mathbf{H}_{m} \mathbf{t}_{n}\right)^{\dagger}+N_{0} \mathbf{I}\right]^{-1} \mathbf{H}_{m} \mathbf{t}_{m},
$$

where $\varsigma_{m}$ is a constant chosen to ensure $\left\|\mathbf{r}_{m}\right\|=1$. As mentioned before, this receiver design will further increase the received SINR, so the actual resulting SINRs are higher than the requirements $\left\{\gamma_{m}\right\}$ which are made achievable even with the imperfect CSIT.

\subsection{Extension to the case with imperfect CSIR}

Here, a more general case is considered where neither CSIT nor CSIR is perfect. In this case, we use $\tilde{\mathbf{H}}_{\mathrm{BF}}^{(m)}$ and $\tilde{\mathbf{H}}_{\mathrm{T}}^{(m)}$ to distinguish the estimated CSIR from CSIT. The details of the uncertainty CSIT and CSIR models are given in Section 2.2. At the mobile user $m$, the actual receive beamforming vector $\mathbf{r}_{m}$ should be optimized based on the knowledge of the estimated CSIR $\widetilde{\mathbf{H}}_{\mathrm{BF}}^{(m)}$ and the uncertainty region $\boldsymbol{U}_{\mathrm{R}}^{(m)}$. To be specific, $\mathbf{r}_{m}$ is chosen to be the solution of the following:

$$
\begin{aligned}
& \max _{\left\|\mathbf{r}_{m}\right\|=1} \min _{\Delta \mathbf{H}_{\mathrm{BF}}^{(m)} \in U_{\mathrm{R}}^{(m)}} F_{m}\left(\Delta \mathbf{H}_{\mathrm{BF}}^{(m)}, \mathbf{r}_{m}\right), \\
& \text { s.t. trace }\left(\Delta \mathbf{H}_{\mathrm{BF}}^{(m)} \mathbf{U}_{\mathrm{R}}^{(m)} \Delta \mathbf{H}_{\mathrm{BF}}^{(m)}{ }^{\dagger}\right) \leq \xi_{\mathrm{R}}^{(m)^{2}},
\end{aligned}
$$


where $F_{m}\left(\Delta \mathbf{H}_{\mathrm{BF}}^{(m)}, \mathbf{r}_{m}\right)$ is defined as follows:

$$
\begin{aligned}
F_{m}\left(\Delta \mathbf{H}_{\mathrm{BF}}^{(m)}, \mathbf{r}_{m}\right) & \\
\triangleq & \left|\mathbf{r}_{m}^{\dagger} \mathbf{H}_{m} \mathbf{t}_{m}\right|^{2}-\gamma_{m}\left(\sum_{\substack{n=1 \\
n \neq m}}^{M}\left|\mathbf{r}_{m}^{\dagger} \mathbf{H}_{m} \mathbf{t}_{n}\right|^{2}+N_{0}\right)^{2} \\
= & \left|\mathbf{r}_{m}^{\dagger} \mathbf{H}_{\mathrm{BF}}^{(m)} \mathbf{a}_{m}\right|^{2}-\gamma_{m}\left(\sum_{\substack{n=1 \\
n \neq m}}^{M}\left|\mathbf{r}_{m}^{\dagger} \mathbf{H}_{\mathrm{BF}}^{(m)} \mathbf{a}_{n}\right|^{2}+N_{0}\right)^{2} \\
= & \mathbf{r}_{m}^{\dagger} \tilde{\mathbf{H}}_{\mathrm{BF}}^{(m)} \mathbf{A}_{m} \tilde{\mathbf{H}}_{\mathrm{BF}}^{(m) \dagger} \mathbf{r}_{m}+\mathbf{r}_{m}^{\dagger} \tilde{\mathbf{H}}_{\mathrm{BF}}^{(m)} \mathbf{A}_{m} \Delta \tilde{\mathbf{H}}_{\mathrm{BF}}^{(m) \dagger} \mathbf{r}_{m} \\
& +\mathbf{r}_{m}^{\dagger} \Delta \tilde{\mathbf{H}}_{\mathrm{BF}}^{(m)} \mathbf{A}_{m} \tilde{\mathbf{H}}_{\mathrm{BF}}^{(m) \dagger} \mathbf{r}_{m}+\mathbf{r}_{m}^{\dagger} \Delta \tilde{\mathbf{H}}_{\mathrm{BF}}^{(m)} \mathbf{A}_{m} \Delta \tilde{\mathbf{H}}_{\mathrm{BF}}^{(m) \dagger} \mathbf{r}_{m}-\gamma_{m} N_{0},
\end{aligned}
$$

where

$$
\mathbf{A}_{m} \triangleq \mathbf{a}_{m} \mathbf{a}_{m}^{\dagger}-\gamma_{m} \sum_{\substack{n=1 \\ n \neq m}}^{M} \mathbf{a}_{n} \mathbf{a}_{n}^{\dagger}
$$

and $\mathbf{a}_{m}$ is an all-zero vector except the $m$ th element being unity.

Similar to the optimization described in Section 4.1.2, (43) becomes

$$
\begin{aligned}
& \max _{\substack{g \\
\mathbf{R}_{m} \geq \mathbf{0} \\
s_{\mathrm{R}}^{(m)} \geq 0}} \\
& \text { s.t. }\left\{\begin{array}{cc}
J & \operatorname{vec}\left(\mathbf{A}_{m} \tilde{\mathbf{H}}_{\mathrm{BF}}^{(m)} \mathbf{R}_{m}\right)^{\dagger} \\
{\left[\begin{array}{cc}
J \\
\operatorname{vec}\left(\mathbf{A}_{m} \tilde{\mathbf{H}}_{\mathrm{BF}}^{(m)} \mathbf{R}_{m}\right) & \mathbf{R}_{m} \otimes \mathbf{A}_{m}+\boldsymbol{s}_{\mathrm{R}}^{(m)} \mathbf{I} \otimes \mathbf{U}_{\mathrm{R}}^{(m)}
\end{array}\right] \geq \mathbf{0},} \\
\operatorname{trace}\left(\mathbf{R}_{m}\right)=1,
\end{array}\right.
\end{aligned}
$$

where $J$ denotes trace $\left(\tilde{\mathbf{H}}_{\mathrm{BF}}^{(m)} \mathbf{A}_{m} \tilde{\mathbf{H}}_{\mathrm{BF}}^{(m) \dagger} \mathbf{R}_{m}\right)-\gamma_{m} N_{0}-s_{\mathrm{R}}^{(m)} \xi_{\mathrm{R}}^{(m)^{2}}-$ $g$.

When (46) returns a higher-rank solution for $\mathbf{R}_{m}$, the optimal rank-1 solution can be extracted by the following.

(i) With the higher-rank $\mathbf{R}_{m}$, (46) is indeed a nonconvex quadratic problem in $\operatorname{vec}\left(\Delta \mathbf{H}_{\mathrm{BF}}^{(m)}\right)$ and the technique in [45] can be used to determine the optimal $\operatorname{vec}\left(\Delta \mathbf{H}_{\mathrm{BF}}^{(m)}\right)$ or the worst-case CSI error matrix, denoted as $\Delta \mathbf{W}_{\mathrm{BF}}^{(m)}$.

(ii) Then, the optimal receive beamforming vector has an MMSE form and is given by

$$
\mathbf{r}_{m}=\varsigma_{m}\left[\mathbf{W}_{\mathrm{BF}}^{(m)} \mathbf{W}_{\mathrm{BF}}^{(m) \dagger}+N_{0} \mathbf{I}\right]^{-1} \mathbf{W}_{\mathrm{BF}}^{(m)} \mathbf{a}_{m},
$$

where $\mathbf{W}_{\mathrm{BF}}^{(m)} \triangleq \widetilde{\mathbf{W}}_{\mathrm{BF}}^{(m)}+\Delta \mathbf{W}_{\mathrm{BF}}^{(m)}$, and $\varsigma_{m}$ is chosen to ensure $\left\|\mathbf{r}_{m}\right\|=1$. Note that this MMSE receiver can be used to decode the signal because it not only maximizes the worst-case SINR but also minimizes the worst-case MSE, which facilitates signal demodulation and decoding.

\section{SIMULATION RESULTS}

\subsection{Setup and assumptions}

Simulations are conducted to assess the performance of the proposed algorithm in Rayleigh flat-fading channels, following $\mathcal{C} \mathcal{N}(0,1)$. Unless explicitly stated, we consider that users have the same target SINR and channel error bounds, that is, $\gamma_{m}=\gamma, \xi_{\mathrm{T}}^{(m)}=\xi, \xi_{\mathrm{R}}^{(m)}=\xi^{\prime}$ for all $m$. Further, for the cases of multiuser MIMO, users are assumed to have an equal number of antennas, that is, $n_{R}^{(m)}=n_{R}$ for all $m$. The notation $M$-user $\left(n_{T}, n_{R}\right)$ will be used to denote an $M$-user MIMO system with $n_{T}$ transmit antennas and $n_{R}$ receive antennas per mobile user. In the simulations, we assume that the CSI error is Gaussian distributed over the bounded uncertainty region with the probability that the Gaussian CSI error falls within the region, to be $99 \%$ for any given bounds $\xi_{T}^{(m)}$ or $\xi_{\mathrm{R}}^{(m)}$.

The average total transmit SNR, defined as $E[P] / N_{0}$, will be regarded as the performance measure. The service probability, which is defined as the probability that a given method gives a feasible solution, will be used to measure the robustness of the method against CSI errors. Several benchmarks are compared with the algorithm proposed in Section 4 . They are as follows.

(i) The "nonrobust" design, which optimizes the users' beamforming vectors based on the estimated CSIT and $\operatorname{CSIR}\left(\left\{\tilde{\mathbf{H}}_{\mathrm{T}}^{(m)}\right\}\right)$. For multiuser MISO, the optimal solution in [14] is applied while the iterative method in [17] will be used for MIMO. The channel uncertainty regions, $\mathcal{U}_{\mathrm{T}}^{(m)}$ and $\boldsymbol{U}_{\mathrm{R}}^{(m)}$, are ignored, so this method is expected to have high probability of outage.

(ii) Optimal power allocation (19) with fixed beamforming vectors, which chooses $\left\{\mathbf{t}_{m}\right\}$ to be the ZF beamforming vectors in [16] and then the power allocation is optimized with these fixed beamforming vectors based on (19).

(iii) Robust solution based on the lower bound (18), which obtains the robust solution by solving (18). Since (18) is of the same form as with perfect CSIT, the method in [14] can be used to find the optimal solution. Note also that we have not derived the SINR lower bound for MIMO systems. Therefore, results of this method are provided only for multiuser MISO systems.

To enable a fair comparison of SNR, our first discussion in the following will be based on the channel and error realizations where all of the methods (both the benchmarks and the proposed one) are feasible. In particular, it implies that for MISO cases, (22) always returns an all-rank-1 solution and thus the proposed method is also the global optimal robust solution. The feasibility issues for various algorithms and their probability of outage will then be evaluated and compared when we conclude this section. 


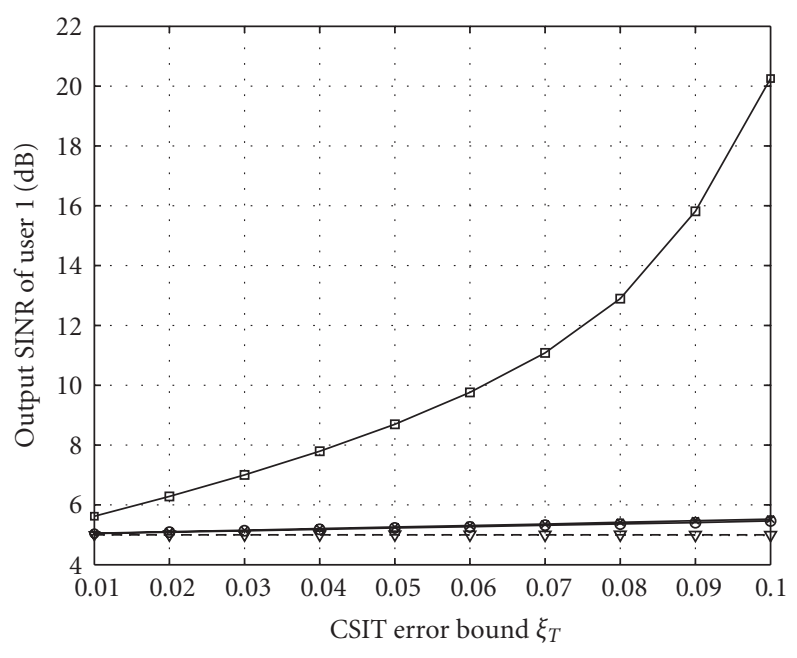

- - - Target SINR

$\rightarrow$ Robust solution using lower bound (13)

- Optimal power-only allocation (14) with ZF

$\rightarrow$ Proposed algorithm/optimal solution

$\nabla \cdots$ Nonrobust design

FIGURE 2: The user's output SINR averaged over channel uncertainty for a given channel realization for various channel error bound for a 3 -user $(3,1)$ system with $\gamma=5(\mathrm{~dB})$.

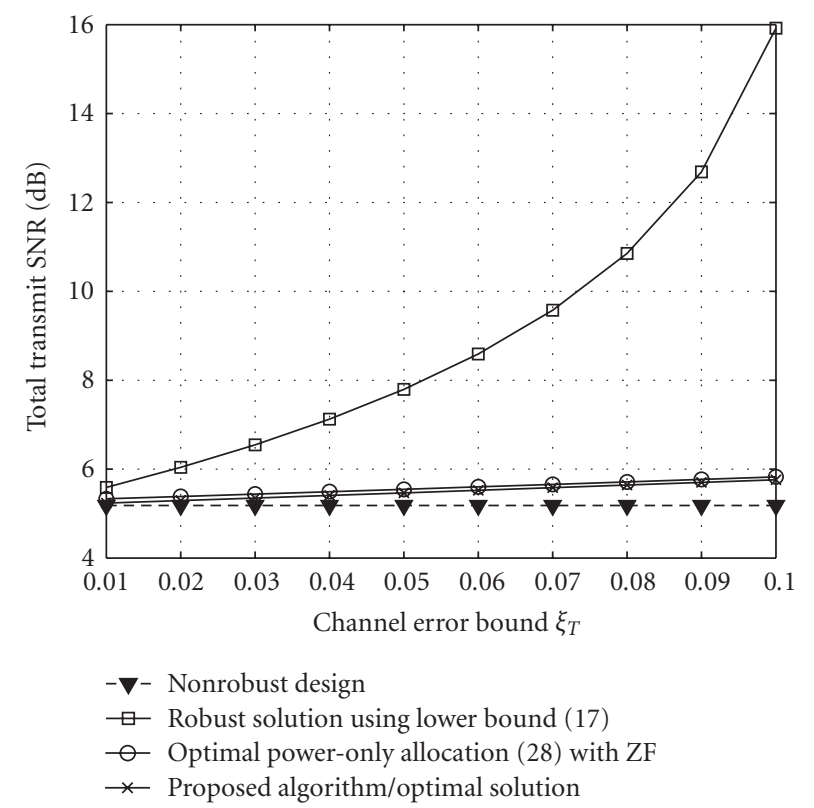

FIgURE 3: The total transmit SNR versus the channel error bound for a 3 -user $(3,1)$ system.

\subsection{Results}

Results in Figures $2-4$ are provided for a 3-user $(3,1)$ system with the CSI uncertainty parameters $\mathbf{U}_{\mathrm{T}}^{(m)}=\mathbf{I}, \xi_{\mathrm{T}}^{(m)}=\xi$, for all $\mathrm{m}$. In Figures 2 and 3, the users' target SINR are set to be $5(\mathrm{~dB})$ while $10(\mathrm{~dB})$ is considered in Figure 4 . Results in the first two figures examine the performance

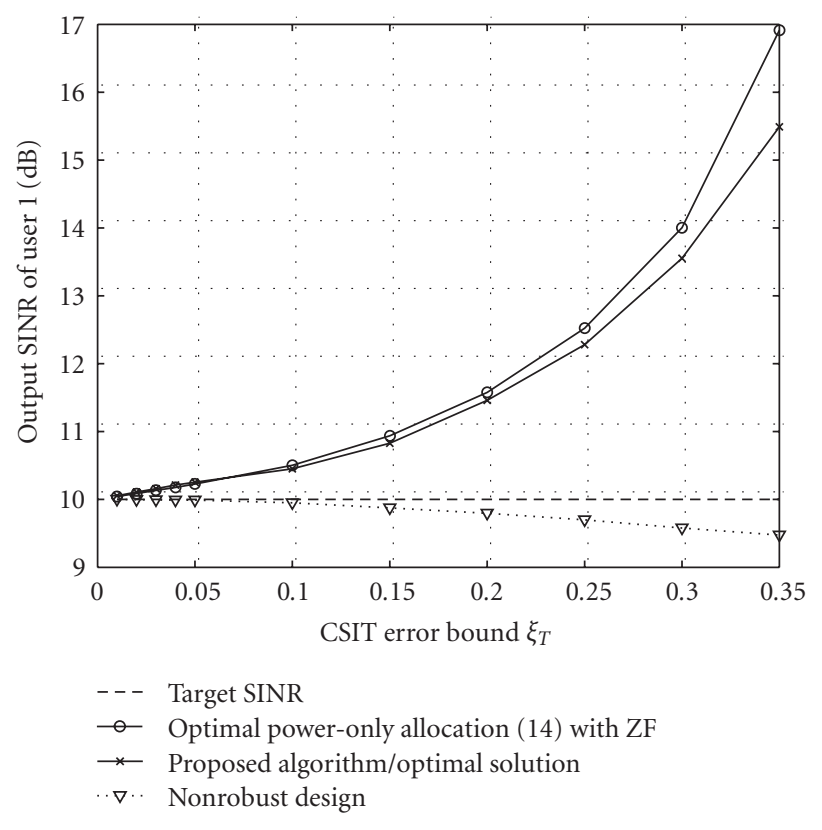

FIGURE 4: The user's output SINR averaged over channel uncertainty for a given channel realization for various channel error bound for a 3 -user $(3,1)$ system with $\gamma=10(\mathrm{~dB})$.

of various schemes with small channel uncertainty bound, up to $\xi=0.1$. Results in Figure 2 show the output SINRs of user 1 for a particular channel realization, averaged over the channel uncertainty. As we can see, the proposed algorithm (which is optimal for MISO) and the optimal power-only allocation achieve slightly greater SINR than the target, which is expected because the optimization is done in a way that the target can still be achieved at the worst error conditions. In addition, results also illustrate that the lower bounding approach achieves much higher SINR than required and this loose bound leads to a huge power penalty for ensuring the required QoS. In particular, results in Figure 3 show that the SNR penalty of the SINR bounding approach (18) grows with the channel error bound and there is an SNR gap of as large as $10(\mathrm{~dB})$ if $\xi=0.1$, as compared to the proposed algorithm and the optimal poweronly allocation. Moreover, results indicate that the optimal joint power and beamforming solution performs similarly to the optimal power-only allocation with fixed beamforming vectors. However, we will soon observe that this is only the case for systems with small number of users, and that the channel is feasible for both solutions. From the transmit SNR point of view, the nonrobust design is always the best but a close observation of the data in Figure 2 reveals that the output SINR is always smaller than the target, meaning that the solution is actually not feasible. This problem becomes much more apparent when $\gamma=10(\mathrm{~dB})$ is considered in Figure 4, and the gap between the output and target SINRs grows farther apart as $\xi$ increases.

In Figure 5, the feasibility regions of the optimal solution, the optimal power-only allocation (19) with ZF beamforming vectors, and the method using (18) are 
TABLE 1: Service probabilities for a 2-user $(2,1)$ system with imperfect CSIT but perfect CSIR.

\begin{tabular}{lllll}
\hline Channel error bound $\xi$ & $\begin{array}{l}\text { Proposed } \\
\text { algorithm } \\
\text { (Section 4.3) }\end{array}$ & $\begin{array}{l}\text { Optimal power } \\
\text { (19) with fixed ZF } \\
\text { vectors } \\
\text { (Section 3.3.3) }\end{array}$ & $\begin{array}{l}\text { Solution using } \\
\text { lower bound (18) } \\
\text { (Section 3.2) }\end{array}$ & Nonrobust design \\
\hline 0.01 & 1 & 1 & 0.82 & 0.24 \\
0.05 & 0.98 & 0.97 & 0.17 & 0.22 \\
0.1 & 0.9 & 0.87 & 0.001 & 0.20 \\
0.15 & 0.78 & 0.71 & 0 & 0.17 \\
0.2 & 0.65 & 0.55 & 0 & 0.15 \\
0.3 & 0.32 & 0.22 & 0 & 0.04 \\
\hline
\end{tabular}

plotted for a particular channel realization $\widetilde{\mathbf{h}}_{1}=[1.2272+$ $0.4176 i 0.1014-0.3508 i], \tilde{\mathbf{h}}_{2}=[-0.8694+1.2169 i$ $0.2530-1.1055 i]$ of a 2 -user $(2,1)$ system. (Note that as these three problems are all convex, they can be optimally solved and the feasibility can also be easily checked using some standard numerical algorithms for convex optimization, such as the interior-point method.) In this figure, $\gamma=5$ $(\mathrm{dB})$ and $\xi=0.05$ are assumed. The vertices indicate the minimum transmit power (or SNR) needed for each scheme. As we can see, the region for the lower bounding approach is the smallest while the region for the optimal solution is the largest and embraces that of the other two schemes. This demonstrates that although previous results have shown that the optimal solution and the optimal power-only allocation perform similarly, there is a detrimental implication on the feasibility by not optimizing the beamforming vectors and the power allocation jointly. This point will further be elucidated later in Table 1.

Results have so far shown that for multiuser MISO systems, the proposed algorithm performs similarly as the power-only optimization with ZF beamforming vectors. This conclusion is however not true for a MIMO system and when the channel uncertainty is more severe, for example, $\xi$ as large as 0.3. These results are shown in Figure 6 for 3-user $(3,2)$ and 4 -user $(4,3)$ systems with $\gamma=10(\mathrm{~dB})$. As we can see, larger gaps in SNR are observed and they grow considerably with the channel error bound $\xi$ and the number of users. In particular, a gap of $8(\mathrm{~dB})$ is observed for a 4 -user system when $\xi=0.3$ while a gap of $7(\mathrm{~dB})$ appears for a 3 -user system with the same level of CSIT uncertainty. Note that the results in this figure are for the cases with both perfect and imperfect CSIR since the transmit SNR depends only on the transmit beamforming vectors, obtained based on CSIT.

The performances of various algorithms when they are all feasible are pretty well addressed now. However, it is also important to know how they actually perform for general random channels and error conditions particularly in terms of their service probability (i.e., the probability that a given method gives a feasible solution with the users' SINR constraints satisfied). Here, we examine this by providing the service (or nonoutage) probabilities for the various algorithms in Tables 1 and 2. Results in the tables illustrate that the proposed algorithm decreases the probability of outage

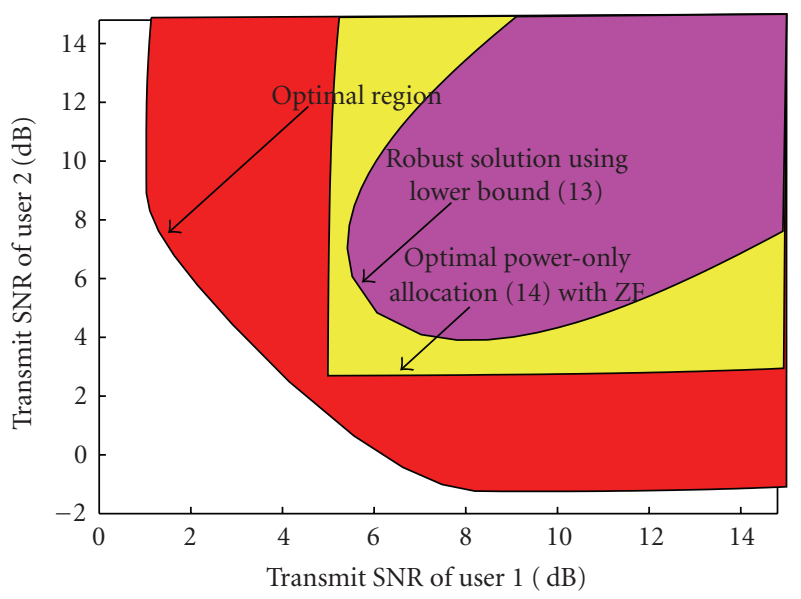

FIGURE 5: The feasibility regions of a 2-user $(2,1)$ system for various robust algorithms for a particular channel realization.

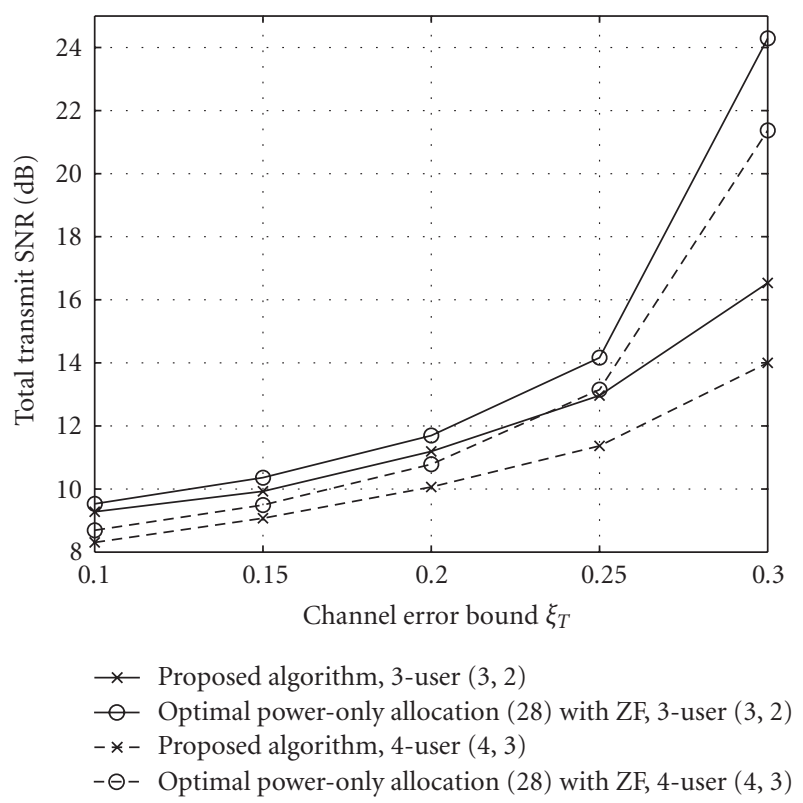

FIgURE 6: The total transmit SNR versus the channel error bound for 3-user $(3,2)$ and 4-user $(4,3)$ systems. 
TABLE 2: Service probabilities for multiuser MIMO systems where both imperfect CSIT and imperfect CSIR are considered such that $\xi_{\mathrm{T}}^{(m)}=\xi$ for all $m, \xi_{\mathrm{R}}^{(m)}=\xi^{\prime}$ for all $m$ and $\xi=2 \xi^{\prime}$.

\begin{tabular}{ccccccc}
\hline $\begin{array}{l}\text { Channel error } \\
\text { bound } \xi\end{array}$ & $\begin{array}{c}\text { Robust 2-user } \\
(2,2)\end{array}$ & $\begin{array}{c}\text { Nonrobust 2-user } \\
(2,2)\end{array}$ & $\begin{array}{c}\text { Robust 3-user } \\
(3,2)\end{array}$ & $\begin{array}{c}\text { Nonrobust 3-user } \\
(3,2)\end{array}$ & $\begin{array}{c}\text { Robust 4-user } \\
(4,3)\end{array}$ & $\begin{array}{c}\text { Nonrobust 4-user } \\
(4,3)\end{array}$ \\
\hline 0.01 & 1 & 0.25 & 1 & 0.12 & 1 & 0.061 \\
0.05 & 1 & 0.23 & 1 & 0.1 & 0.085 & 1 \\
0.1 & 1 & 0.21 & 0.99 & 0.066 & 0.99 & 0.035 \\
0.15 & 0.99 & 0.19 & 0.99 & 0.053 & 0.99 & 0.03 \\
0.2 & 0.98 & 0.18 & 0.98 & 0.034 & 0.98 & 0.01 \\
0.3 & 0.84 & 0.14 & 0.77 & & 0.02 \\
\hline
\end{tabular}

TABLe 3: The probability, $\mathcal{P}$, that the proposed algorithm (22) does not give an all-rank-1 solution. In this table, $*$ means that (22) does not even have a feasible solution.

\begin{tabular}{|c|c|c|c|c|}
\hline 3 -user $(3,1)$ system & Ellipsoid & & Nonellipsoid & \\
\hline $\operatorname{Ranks}$ of $\left(\mathbf{U}_{\top}^{(1)}, \mathbf{U}_{\top}^{(2)}, \mathbf{U}_{\top}^{(3)}\right)$ & $(3,3,3)$ & $(3,3,2)$ & $(3,2,2)$ & $(3,1,1)$ \\
\hline $\mathcal{P}$ & 0.0226 & 1 & 1 & $*$ \\
\hline 4 -user $(4,1)$ system & Ellipsoid & & Nonellipsoid & \\
\hline $\operatorname{Ranks}$ of $\left(\mathbf{U}_{\top}^{(1)}, \mathbf{U}_{\top}^{(2)}, \mathbf{U}_{\top}^{(3)}, \mathbf{U}_{\top}^{(4)}\right)$ & $(4,4,4,4)$ & $(4,3,3,2)$ & $(4,3,2,1)$ & $(4,2,1,1)$ \\
\hline $\mathcal{P}$ & 0.0274 & 1 & 1 & $*$ \\
\hline 5-user $(5,1)$ system & Ellipsoid & & Nonellipsoid & \\
\hline $\operatorname{Ranks}$ of $\left(\mathbf{U}_{\top}^{(1)}, \mathbf{U}_{\top}^{(2)}, \mathbf{U}_{\top}^{(3)}, \mathbf{U}_{\top}^{(4)}\right)$ & $(5,5,5,5,5)$ & $(5,4,4,3,2)$ & $(5,4,3,3,2)$ & $(5,4,3,2,1)$ \\
\hline $\mathcal{P}$ & 0.0296 & 1 & 1 & $*$ \\
\hline
\end{tabular}

by orders of magnitude, when compared to the nonrobust design. Besides, there is a remarkable increase in the service probability by using the proposed algorithm over the optimal power-only allocation. On the other hand, however, if $\xi$ is too large, the problem itself is more likely to become infeasible (and there exists no robust solution), leading to an unacceptably low service probability. In addition, we can see that for a given channel error bound $\xi$, multiuser MIMO has a much higher service probability than multiuser MISO even if imperfect CSIR is considered for the MIMO cases.

In Table 3, the tightness of the relaxation approach is examined and the probability that the proposed algorithm (22) does not give an all-rank-1 solution is shown, which is designated as $\mathcal{P}$. In the simulations, $\xi=0.1$ and random SINR requirements are considered. The results are obtained by averaging over $10^{5}$ independent channel realizations and $\left\{\mathbf{U}_{\mathrm{T}}^{(m)}\right\}$. It is observed that with full-rank matrices $\left\{\mathbf{U}_{\mathrm{T}}^{(m)}\right\}$ (i.e., the CSI error regions are ellipsoids), the rank-1 solution exists with high probability, while with nonfull-rank $\left\{\mathbf{U}_{\mathrm{T}}^{(m)}\right\}$ (i.e., nonellipsoids), the proposed algorithm always outputs a higher-rank solution and in some cases, the problem (22) is even infeasible.

\section{CONCLUSION}

This paper has studied the worst-case robust beamforming design of a downlink multiuser MIMO antenna system where the CSIT and CSIR are known but imperfect, and the channel uncertainty regions, which are modeled by ellipsoids, are also known. With an aim to minimizing the overall transmit power while ensuring that the users' target SINRs for all possible channel uncertainty conditions within the ellipsoids, this paper has presented techniques to jointly optimize the power allocation and the transmitreceive beamforming vectors for the users, based on the imperfect CSIT, the perfect or imperfect CSIR, and the channel uncertainty regions. Using S-procedure and rank relaxation, it is possible to obtain the globally optimal joint robust power and beamforming solution for a multiuser MISO system while a convergent iterative algorithm has been proposed to obtain a suboptimal robust solution for a multiuser MIMO system. Simulation results have demonstrated that the proposed algorithm yields a significant power reduction to ensure robustness than an SINR bounding approach, and the optimal power-only allocation with fixed ZF beamforming vectors. The proposed algorithm has also been shown to have the largest feasibility region and yield the least outage probability as compared to other robust and nonrobust schemes.

\section{APPENDIX}

\section{A. DERIVATION OF THE KARUSH-KUHN-TUCKER (KKT) CONDITIONS FOR PROBLEM (22)}

Introducing the dual variables for all $m$,

$$
\begin{gathered}
\lambda_{m}, r_{\mathrm{T}}^{(m)}, r_{\mathrm{R}}^{(m)} \geq 0, \\
\mathbf{W}_{m} \triangleq\left[\begin{array}{cc}
\lambda_{m} & \mathbf{v}_{m}^{\dagger} \\
\mathbf{v}_{m} & \mathbf{V}_{m}
\end{array}\right], \quad \mathbf{S}_{m} \geq \mathbf{0},
\end{gathered}
$$


the Lagrangian of (22) is given by

$$
\begin{aligned}
\mathcal{L} & \left(\left\{\mathbf{T}_{m}\right\}, s_{\mathrm{T}}^{(m)}, s_{\mathrm{R}}^{(m)}, \mathbf{S}_{m}, r_{\mathrm{T}}^{(m)}, r_{\mathrm{R}}^{(m)}, \mathbf{W}_{m}\right) \\
= & \sum_{m=1}^{M}\left[\operatorname{trace}\left(\mathbf{T}_{m}\right)-\operatorname{trace}\left(\mathbf{T}_{m} \mathbf{S}_{m}\right)\right]-\sum_{m=1}^{M}\left(r_{\mathrm{T}}^{(m)} s_{\mathrm{T}}^{(m)}+r_{\mathrm{R}}^{(m)} s_{\mathrm{R}}^{(m)}\right) \\
& -\sum_{m=1}^{M} \operatorname{trace}\left(\left[\begin{array}{cc}
K & \tilde{\mathbf{h}}_{m} \mathbf{Q}_{m} \\
\mathbf{Q}_{m} \tilde{\mathbf{h}}_{m}^{\dagger} & \mathbf{Q}_{m}+s_{\mathrm{T}}^{(m)} \mathbf{U}_{\mathrm{T}}^{(m)}
\end{array}\right] \mathbf{W}_{m}\right),
\end{aligned}
$$

where $K$ denotes $\tilde{\mathbf{h}}_{m} \mathbf{Q}_{m} \tilde{\mathbf{h}}_{m}^{\dagger}-\gamma_{m} N_{0}-s_{\mathrm{T}}^{(m)} \xi_{\mathrm{T}}^{(m)^{2}}$.

Using the first-order optimality conditions

$$
\begin{aligned}
\frac{\partial \mathcal{L}}{\partial s_{\mathrm{T}}^{(m)}}= & -r_{\mathrm{T}}^{(m)}+\lambda_{m} \xi_{\mathrm{T}}^{(m)^{2}}-\operatorname{trace}\left(\mathbf{U}_{\mathrm{T}}^{(m)} \mathbf{V}_{m}\right)=0, \\
\frac{\partial \mathcal{L}}{\partial s_{\mathrm{R}}^{(m)}}= & -r_{\mathrm{R}}^{(m)}+\lambda_{m} \xi_{\mathrm{R}}^{(m)^{2}}-\operatorname{trace}\left(\mathbf{U}_{\mathrm{R}}^{(m)} \mathbf{V}_{m}\right)=0, \\
\frac{\partial \mathcal{L}}{\partial \mathbf{T}_{m}}= & \mathbf{I}-\mathbf{S}_{m}-\left(\lambda_{m} \tilde{\mathbf{h}}_{m}^{\dagger} \tilde{\mathbf{h}}_{m}+\mathbf{v}_{m} \tilde{\mathbf{h}}_{m}+\tilde{\mathbf{h}}_{m}^{\dagger} \mathbf{v}_{m}^{\dagger}+\mathbf{V}_{m}\right) \\
& +\sum_{\substack{n=1 \\
n \neq m}}^{M} \gamma_{n}\left(\lambda_{n} \tilde{\mathbf{h}}_{n}^{\dagger} \tilde{\mathbf{h}}_{n}+\mathbf{v}_{n} \tilde{\mathbf{h}}_{n}+\tilde{\mathbf{h}}_{n}^{\dagger} \mathbf{v}_{n}^{\dagger}+\mathbf{V}_{n}\right)=\mathbf{0},
\end{aligned}
$$

we can obtain the following dual problem

$$
\begin{aligned}
& \max _{\left\{\lambda_{m}, \mathbf{V}_{m}, \mathbf{v}_{m}\right\}_{m}^{M}} \sum_{m=1}^{M} \lambda_{m} \gamma_{m} N_{0} \\
& \text { s.t. }\left\{\begin{array}{l}
\lambda_{m} \xi_{\mathrm{T}}^{(m)^{2}}-\operatorname{trace}\left(\mathbf{U}_{\mathrm{T}}^{(m)} \mathbf{V}_{m}\right) \geq 0, \\
\lambda_{m} \xi_{\mathrm{R}}^{(m)^{2}}-\operatorname{trace}\left(\mathbf{U}_{\mathrm{R}}^{(m)} \mathbf{V}_{m}\right) \geq 0, \\
\mathbf{I}-\left(\lambda_{m} \tilde{\mathbf{h}}_{m}^{\dagger} \tilde{\mathbf{h}}_{m}+\mathbf{v}_{m} \tilde{\mathbf{h}}_{m}+\widetilde{\mathbf{h}}_{m}^{\dagger} \mathbf{v}_{m}^{\dagger}+\mathbf{V}_{m}\right) \\
\quad+\sum_{\substack{n=1 \\
n \neq m}}^{M} \gamma_{n}\left(\lambda_{n} \tilde{\mathbf{h}}_{n}^{\dagger} \tilde{\mathbf{h}}_{n}+\mathbf{v}_{n} \tilde{\mathbf{h}}_{n}+\tilde{\mathbf{h}}_{n}^{\dagger} \mathbf{v}_{n}^{\dagger}+\mathbf{V}_{n}\right) \succeq \mathbf{0} \\
{\left[\begin{array}{cc}
\lambda_{m} & \mathbf{v}_{m}^{\dagger} \\
\mathbf{v}_{m} & \mathbf{V}_{m}
\end{array}\right] \succeq \mathbf{0 .}}
\end{array}\right.
\end{aligned}
$$

\section{ACKNOWLEDGMENTS}

This work was supported in part by The Hong Kong Research Grants Council under Grant HKU7175/03E and The University Research Committee of The University of Hong Kong, and in part by The Engineering and Physical Science Research Council (EPSRC) under Grant EP/D058716/1.

\section{REFERENCES}

[1] T. S. Rappaport, Wireless Communications: Principles and Practice, Prentice Hall, Upper Saddle River, NJ, USA, 1996.

[2] G. J. Foschini and M. J. Gans, "On limits of wireless communications in a fading environment when using multiple antennas," Wireless Personal Communications, vol. 6, no. 3, pp. 311-335, 1998.
[3] I. E. Telatar, "Capacity of multi-antenna Gaussian channels," Tech. Rep., AT \& T Bell Labs, Murray Hill, NJ, USA, 1995.

[4] V. Tarokh, N. Seshadri, and A. R. Calderbank, "Space-time codes for high data rate wireless communication: performance criterion and code construction," IEEE Transactions on Information Theory, vol. 44, no. 2, pp. 744-765, 1998.

[5] A. Goldsmith, S. A. Jafar, N. Jindal, and S. Vishwanath, "Capacity limits of MIMO channels," IEEE Journal on Selected Areas in Communications, vol. 21, no. 5, pp. 684-702, 2003.

[6] S. M. Alamouti, "A simple transmit diversity technique for wireless communications," IEEE Journal on Selected Areas in Communications, vol. 16, no. 8, pp. 1451-1458, 1998.

[7] G. G. Raleigh and J. M. Cioffi, "Spatio-temporal coding for wireless communication," IEEE Transactions on Communications, vol. 46, no. 3, pp. 357-366, 1998.

[8] K.-K. Wong, R. S. K. Cheng, K. Ben Letaief, and R. D. Murch, "Adaptive antennas at the mobile and base stations in an OFDM/TDMA system," IEEE Transactions on Communications, vol. 49, no. 1, pp. 195-206, 2001.

[9] M. Costa, "Writing on dirty paper," IEEE Transactions on Information Theory, vol. 29, no. 3, pp. 439-441, 1983.

[10] H. Weingarten, Y. Steinberg, and S. Shamai, "The capacity region of the Gaussian multiple-input multiple-output broadcast channel," IEEE Transactions on Information Theory, vol. 52, no. 9, pp. 3936-3964, 2006.

[11] S. Vishwanath, N. Jindal, and A. Goldsmith, "Duality, achievable rates, and sum-rate capacity of Gaussian MIMO broadcast channels," IEEE Transactions on Information Theory, vol. 49, no. 10, pp. 2658-2668, 2003.

[12] W. Yu and J. M. Cioffi, "Sum capacity of Gaussian vector broadcast channels," IEEE Transactions on Information Theory, vol. 50, no. 9, pp. 1875-1892, 2004.

[13] F. Rashid-Farrokhi, K. J. R. Liu, and L. Tassiulas, "Transmit beamforming and power control for cellular wireless systems," IEEE Journal on Selected Areas in Communications, vol. 16, no. 8, pp. 1437-1450, 1998.

[14] M. Bengtsson and B. Ottersten, "Optimal and suboptimal transmit beamforming," in Handbook of Antennas in Wireless Communications, CRC Press, Boca Raton, Fla, USA, 2001.

[15] K.-K. Wong, R. D. Murch, and K. B. Letaief, "Performance enhancement of multiuser MIMO wireless communication systems," IEEE Transactions on Communications, vol. 50, no. 12, pp. 1960-1970, 2002.

[16] Z. Pan, K.-K. Wong, and T.-S. Ng, "Generalized multiuser orthogonal space-division multiplexing," IEEE Transactions on Wireless Communications, vol. 3, no. 6, pp. 1969-1973, 2004.

[17] G. Zheng, K.-K. Wong, and T.-S. Ng, "Convergence analysis of downlink MIMO antenna systems using second-order cone programming," in Proceedings of the 62nd IEEE Vehicular Technology Conference (VTC '05), vol. 1, pp. 492-496, Dallas, Tex, USA, September 2005.

[18] M. Schubert and H. Boche, "Solution of the multiuser downlink beamforming problem with individual SINR constraints," IEEE Transactions on Vehicular Technology, vol. 53, no. 1, pp. 18-28, 2004.

[19] A. Wiesel, Y. C. Eldar, and S. Shamai, "Linear precoding via conic optimization for fixed MIMO receivers," IEEE Transactions on Signal Processing, vol. 54, no. 1, pp. 161-176, 2006.

[20] J. Diaz, Z. Latinovic, and Y. Bar-Ness, "Impact of imperfect channel state information upon the outage capacity of rayleigh fading channels," in Proceedings of IEEE Global Telecommunications Conference (GLOBECOM '04), vol. 2, pp. 887-892, Dallas, Tex, USA, November-December 2004. 
[21] T. Yoo and A. Goldsmith, "Capacity and power allocation for fading MIMO channels with channel estimation error," IEEE Transactions on Information Theory, vol. 52, no. 5, pp. 22032214, 2006.

[22] A. Vakili, M. Sharif, and B. Hassibi, "The effect of channel estimation error on the throughput of broadcast channels," in Proceedings of IEEE International Conference on Acoustics, Speech and Signal Processing (ICASSP '06), vol. 4, pp. 29-32, Toulouse, France, May 2006.

[23] N. Jindal, "MIMO broadcast channels with finite-rate feedback," IEEE Transactions on Information Theory, vol. 52, no. 11, pp. 5045-5060, 2006.

[24] H. Cox, R. M. Zeskind, and M. M. Owen, "Robust adaptive beamforming," IEEE Transactions on Signal Processing, vol. 35, no. 10 , pp. 1365-1376, 1987.

[25] K. L. Bell, Y. Ephraim, and H. L. Van Trees, "A Bayesian approach to robust adaptive beamforming," IEEE Transactions on Signal Processing, vol. 48, no. 2, pp. 386-398, 2000.

[26] D. P. Palomar, A unified framework for communications through MIMO channels, Ph.D. dissertation, Technical University of Catalonia, Barcelona, Spain, May 2003.

[27] A. Pascual-Iserte, D. P. Palomar, A. I. Pérez-Neira, and M. Á. Lagunas, "A robust maximin approach for MIMO communications with imperfect channel state information based on convex optimization," IEEE Transactions on Signal Processing, vol. 54, no. 1, pp. 346-360, 2006.

[28] A. Wiesel, Y. C. Eldar, and S. Shamai, "Optimization of the MIMO compound capacity," IEEE Transactions on Wireless Communications, vol. 6, no. 3, pp. 1094-1101, 2007.

[29] J. Wolfowitz, Coding Theorems of Information Theory, Springer, Berlin, Germany, 3rd edition, 1978.

[30] S. A. Vorobyov, A. B. Gershman, and Z.-Q. Luo, "Robust adaptive beamforming using worst-case performance optimization: a solution to the signal mismatch problem," IEEE Transactions on Signal Processing, vol. 51, no. 2, pp. 313-324, 2003.

[31] M. Biguesh, S. Shahbazpanahi, and A. B. Gershman, "Robust downlink power control in wireless cellular systems," EURASIP Journal on Wireless Communications and Networking, vol. 2004, no. 2, pp. 261-272, 2004.

[32] M. Payaró, Impact of channel state information on the analysis and design of multiantenna communicationv systems, Ph.D. dissertation, Technical University of Catalonia, Barcelona, Spain, February 2007.

[33] M. Payaró, A. Pascual-Iserte, and M. Á. Lagunas, "Robust power allocation designs for multiuser and multiantenna downlink communication systems through convex optimization," IEEE Journal on Selected Areas in Communications, vol. 25, no. 7, pp. 1390-1401, 2007.

[34] M. B. Shenouda and T. N. Davidson, "Linear matrix inequality formulations of robust QoS precoding for broadcast channels," in Proceedings of the Canadian Conference on Electrical and Computer Engineering (CCECE '07), pp. 324328, Vancouver, Canada, April 2007.

[35] M. B. Shenouda and T. N. Davidson, "Convex conic formulations of robust downlink precoder designs with quality of service constraints," IEEE Journal on Selected Topics in Signal Processing, vol. 1, no. 4, pp. 714-724, 2007.

[36] A. L. Fradkov and V. A. Yakubovich, "The S-procedure and the duality relation in convex quadratic programming problems," Vestnik Leningrad University: Mathematics, vol. 155, no. 1, pp. 81-87, 1973.

[37] S. Boyd and L. Vandenberghe, Convex Optimization, Cambridge University Press, Cambridge, UK, 2004.
[38] W. Rhee, W. Yu, and J. Cioff, "The optimality of beamforming in uplink multiuser wireless systems," IEEE Transactions on Wireless Communications, vol. 3, no. 1, pp. 86-96, 2004.

[39] T. Yoo and A. Goldsmith, "On the optimality of multiantenna broadcast scheduling using zero-forcing beamforming," IEEE Journal on Selected Areas in Communications, vol. 24, no. 3, pp. 528-541, 2006.

[40] A. Bayesteh and A. K. Khandani, "On the user selection for MIMO broadcast channels," in Proceedings of IEEE International Symposium on Information Theory (ISIT '05), pp. 23252329, Adelaide, Australia, September 2005.

[41] F. Boccardi and H. Huang, "A near-optimum technique using linear precoding for the MIMO broadcast channel," in Proceedings of IEEE International Conference on Acoustics, Speech and Signal Processing (ICASSP '07), vol. 3, pp. 17-20, Honolulu, Hawaii, USA, April 2007.

[42] D. Bertsimas and M. Sim, "Tractable approximations to robust conic optimization problems," Mathematical Programming, vol. 107, no. 1-2, pp. 5-36, 2006.

[43] I. Pólik and T. Terlaky, "A survey of the S-lemma," SIAM Review, vol. 49, no. 3, pp. 371-418, 2007.

[44] A. Ben-Tal and A. S. Nemirovskii, Lectures on Modern Convex Optimization, Society for Industrial and Applied Mathematics, Philadelphia, Pa, USA, 2001.

[45] A. Beck and Y. C. Eldar, "Strong duality in nonconvex quadratic optimization with two quadratic constraints," SIAM Journal on Optimization, vol. 17, no. 3, pp. 844-860, 2006.

[46] R. A. Monzingo and T. W. Miller, Introduction to Adaptive Arrays, John Wiley \& Sons, New York, NY, USA, 1980. 